\title{
The Mann-Type Extragradient Iterative Algorithms with Regularization for Solving Variational Inequality Problems, Split Feasibility, and Fixed Point Problems
}

\author{
Lu-Chuan Ceng, ${ }^{1}$ Himanshu Gupta, ${ }^{2}$ and Ching-Feng Wen ${ }^{3}$ \\ ${ }^{1}$ Department of Mathematics, Shanghai Normal University and Scientific Computing Key Laboratory of Shanghai Universities, \\ Shanghai 200234, China \\ ${ }^{2}$ Department of Mathematics, Aligarh Muslim University, Aligarh 202 002, India \\ ${ }^{3}$ Center for Fundamental Science, Kaohsiung Medical University, Kaohsiung 807, Taiwan
}

Correspondence should be addressed to Ching-Feng Wen; cfwen@kmu.edu.tw

Received 3 December 2012; Accepted 31 December 2012

Academic Editor: Jen-Chih Yao

Copyright $\odot 2013 \mathrm{Lu}$-Chuan Ceng et al. This is an open access article distributed under the Creative Commons Attribution License, which permits unrestricted use, distribution, and reproduction in any medium, provided the original work is properly cited.

\begin{abstract}
The purpose of this paper is to introduce and analyze the Mann-type extragradient iterative algorithms with regularization for finding a common element of the solution set $\Xi$ of a general system of variational inequalities, the solution set $\Gamma$ of a split feasibility problem, and the fixed point set $\operatorname{Fix}(S)$ of a strictly pseudocontractive mapping $S$ in the setting of the Hilbert spaces. These iterative algorithms are based on the regularization method, the Mann-type iteration method, and the extragradient method due to Nadezhkina and Takahashi (2006). Furthermore, we prove that the sequences generated by the proposed algorithms converge weakly to an element of $\operatorname{Fix}(S) \cap \Xi \cap \Gamma$ under mild conditions.
\end{abstract}

\section{Introduction}

Let $\mathscr{H}$ be a real Hilbert space with inner product $\langle\cdot, \cdot\rangle$ and norm $\|\cdot\|$. Let $C$ be a nonempty closed convex subset of $\mathscr{H}$. The projection (nearest point or metric projection) of $\mathscr{H}$ onto $C$ is denoted by $P_{C}$. Let $S: C \rightarrow C$ be a mapping and $\operatorname{Fix}(S)$ be the set of fixed points of $S$. For a given nonlinear operator $A: C \rightarrow \mathscr{H}$, we consider the following variational inequality problem (VIP) of finding $x^{*} \in C$ such that

$$
\left\langle A x^{*}, x-x^{*}\right\rangle \geq 0, \quad \forall x \in C .
$$

The solution set of VIP (1) is denoted by $\operatorname{VI}(C, A)$. The theory of variational inequalities has been studied quite extensively and has emerged as an important tool in the study of a wide class of problems from mechanics, optimization, engineering, science, and social sciences. It is well known that the VIP is equivalent to a fixed point problem. This alternative formulation has been used to suggest and analyze projection iterative method for solving variational inequalities under the conditions that the involved operator must be strongly monotone and Lipschitz continuous. In the recent past, several people have studied and proposed several iterative methods to find a solution of variational inequalities which is also a fixed point of a nonexpansive mapping or strict pseudocontractive mapping; see, for example, [1-9] and the references therein.

For finding an element of $\operatorname{Fix}(S) \cap \operatorname{VI}(C, A)$ when $C$ is closed and convex, $S$ is nonexpansive, and $A$ is $\alpha$-inverse strongly monotone, Takahashi and Toyoda [10] introduced the following Mann-type iterative algorithm:

$$
x_{n+1}=\alpha_{n} x_{n}+\left(1-\alpha_{n}\right) S P_{C}\left(1-\lambda_{n} A x_{n}\right), \quad \forall n \geq 0,
$$

where $P_{C}$ is the metric projection of $\mathscr{H}$ onto $C, x_{0}=x \in$ $C,\left\{\alpha_{n}\right\}$ is a sequence in $(0,1)$, and $\left\{\lambda_{n}\right\}$ is a sequence in $(0,2 \alpha)$. They showed that if $\operatorname{Fix}(S) \cap \operatorname{VI}(C, A) \neq \emptyset$, then the sequence $\left\{x_{n}\right\}$ converges weakly to some $z \in \operatorname{Fix}(S) \cap \operatorname{VI}(C, A)$. Nadezhkina and Takahashi [9] and Zeng and Yao [8] proposed extragradient methods motivated by Korpelevič [11] for finding a common element of the fixed point set of a nonexpansive mapping and the solution set of a variational inequality problem. Further, these iterative methods are 
extended in [12] to develop a new iterative method for finding elements in $\operatorname{Fix}(S) \cap \mathrm{VI}(C, A)$.

Let $B_{1}, B_{2}: C \rightarrow \mathscr{H}$ be two mappings. Recently, Ceng et al. [4] introduced and considered the following problem of finding $\left(x^{*}, y^{*}\right) \in C \times C$ such that

$$
\begin{array}{ll}
\left\langle\mu_{1} B_{1} y^{*}+x^{*}-y^{*}, x-x^{*}\right\rangle \geq 0, & \forall x \in C, \\
\left\langle\mu_{2} B_{2} x^{*}+y^{*}-x^{*}, x-y^{*}\right\rangle \geq 0, & \forall x \in C,
\end{array}
$$

which is called a general system of variational inequalities (GSVI), where $\mu_{1}>0$ and $\mu_{2}>0$ are two constants. The set of solutions of problem (3) is denoted by $\operatorname{GSVI}\left(C, B_{1}, B_{2}\right)$. In particular, if $B_{1}=B_{2}$, then problem (3) reduces to the new system of variational inequalities (NSVI), introduced and studied by Verma [13]. Further, if $x^{*}=y^{*}$, then the NSVI reduces to VIP (1).

Recently, Ceng et al. [4] transformed problem (3) into a fixed point problem in the following way.

Lemma 1 (see [4]). For given $\bar{x}, \bar{y} \in C,(\bar{x}, \bar{y})$ is a solution of problem (3) if and only if $\bar{x}$ is a fixed point of the mapping $G: C \rightarrow C$ defined by

$$
\begin{array}{r}
G(x)=P_{C}\left[P_{C}\left(x-\mu_{2} B_{2} x\right)-\mu_{1} B_{1} P_{C}\left(x-\mu_{2} B_{2} x\right)\right], \\
\forall x \in C,
\end{array}
$$

where $\bar{y}=P_{C}\left(\bar{x}-\mu_{2} B_{2} \bar{x}\right)$.

In particular, if the mapping $B_{i}: C \rightarrow \mathscr{H}$ is $\beta_{i}$-inverse strongly monotone for $i=1,2$, then the mapping $G$ is nonexpansive provided $\mu_{i} \in\left(0,2 \beta_{i}\right)$ for $i=1,2$.

Utilizing Lemma 1, they introduced and studied a relaxed extragradient method for solving GSVI (3).

Throughout this paper, unless otherwise specified, the set of fixed points of the mapping $G$ is denoted by $\Xi$. Based on the relaxed extragradient method and viscosity approximation method, Yao et al. [7] proposed and analyzed an iterative algorithm for finding a common solution of GSVI (3) and fixed point problem of a strictly pseudocontractive mapping $S: C \rightarrow C$, where $C$ is a nonempty bounded closed convex subset of a real Hilbert space $\mathscr{H}$.

Subsequently, Ceng at al. [14] further presented and analyzed an iterative scheme for finding a common element of the solution set of VIP (1), the solution set of GSVI (3), and fixed point set of a strictly pseudocontractive mapping $S: C \rightarrow C$.

Theorem 2 (see [14, Theorem 3.1]). Let $C$ be a nonempty closed convex subset of a real Hilbert space $\mathscr{H}$. Let $A: C \rightarrow \mathscr{H}$ be $\alpha$-inverse strongly monotone, and let $B_{i}: C \rightarrow \mathscr{H}$ be $\beta_{i}$-inverse strongly monotone for $i=1,2$. Let $S: C \rightarrow C$ be a $k$-strictly pseudocontractive mapping such that $\operatorname{Fix}(S) \cap$ $\Xi \cap \operatorname{VI}(C, A) \neq \emptyset$. Let $Q: C \rightarrow C$ be a $\rho$-contraction with $\rho \in[0,1 / 2)$. For given $x_{0} \in C$ arbitrarily, let the sequences $\left\{x_{n}\right\},\left\{y_{n}\right\}$, and $\left\{z_{n}\right\}$ be generated iteratively by

$$
\begin{aligned}
z_{n}= & P_{C}\left(x_{n}-\lambda_{n} A x_{n}\right), \\
y_{n}= & \alpha_{n} Q x_{n} \\
& +\left(1-\alpha_{n}\right) P_{C}\left[P_{C}\left(z_{n}-\mu_{2} B_{2} z_{n}\right)\right. \\
& \left.\quad-\mu_{1} B_{1} P_{C}\left(z_{n}-\mu_{2} B_{2} z_{n}\right)\right], \\
& \quad+\delta_{n} S y_{n}, \quad \forall n \geq 0,
\end{aligned}
$$

where $\mu_{i} \in\left(0,2 \beta_{i}\right)$ for $i=1,2,\left\{\lambda_{n}\right\} \subset(0,2 \alpha]$ and $\left\{\alpha_{n}\right\},\left\{\beta_{n}\right\}$, $\left\{\gamma_{n}\right\},\left\{\delta_{n}\right\} \subset[0,1]$ such that

(i) $\beta_{n}+\gamma_{n}+\delta_{n}=1$ and $\left(\gamma_{n}+\delta_{n}\right) k \leq \gamma_{n}$ for all $n \geq 0$;

(ii) $\lim _{n \rightarrow \infty} \alpha_{n}=0$ and $\sum_{n=0}^{\infty} \alpha_{n}=\infty$;

(iii) $0<\liminf _{n \rightarrow \infty} \beta_{n} \leq \limsup _{n \rightarrow \infty} \beta_{n}<1$ and $\liminf _{n \rightarrow \infty} \delta_{n}>0$;

(iv) $\lim _{n \rightarrow \infty}\left(\gamma_{n+1} /\left(1-\beta_{n+1}\right)-\gamma_{n} /\left(1-\beta_{n}\right)\right)=0$;

(v) $0<\liminf _{n \rightarrow \infty} \lambda_{n} \leq \limsup _{n \rightarrow \infty} \lambda_{n}<2 \alpha$ and $\lim _{n \rightarrow \infty}\left|\lambda_{n+1}-\lambda_{n}\right|=0$.

Then the sequence $\left\{x_{n}\right\}$ generated by (5) converges strongly to $\bar{x}=P_{\operatorname{Fix}(S) \cap \Xi \cap \operatorname{VI}(C, A)} Q \bar{x}$ and $(\bar{x}, \bar{y})$ is a solution of GSVI (3), where $\bar{y}=P_{C}\left(\bar{x}-\mu_{2} B_{2} \bar{x}\right)$.

On the other hand, let $C$ and $Q$ be nonempty closed convex subsets of real Hilbert spaces $\mathscr{H}_{1}$ and $\mathscr{H}_{2}$, respectively. The split feasibility problem (SFP) is to find a point $x^{*}$ with the following property:

$$
x^{*} \in C, \quad A x^{*} \in Q,
$$

where $A \in B\left(\mathscr{H}_{1}, \mathscr{H}_{2}\right)$ and $B\left(\mathscr{H}_{1}, \mathscr{H}_{2}\right)$ denotes the family of all bounded linear operators from $\mathscr{H}_{1}$ to $\mathscr{H}_{2}$.

In 1994, the SFP was first introduced by Censor and Elfving [15], in finite-dimensional Hilbert spaces, for modeling inverse problems which arise from phase retrievals and in medical image reconstruction. A number of image reconstruction problems can be formulated as the SFP; see, for example, [16] and the references therein. Recently, it is found that the SFP can also be applied to study intensitymodulated radiation therapy; see, for example, [17-19] and the references therein. In the recent past, a wide variety of iterative methods have been used in signal processing and image reconstruction and for solving the SFP; see, for example, [16-26] and the references therein. A special case of the SFP is the following convex constrained linear inverse problem [27] of finding an element $x$ such that

$$
x \in C, \quad A x=b .
$$

It has been extensively investigated in the literature using the projected Landweber iterative method [28]. Comparatively, the SFP has received much less attention so far, due to the complexity resulting from the set $Q$. Therefore, whether various versions of the projected Landweber iterative method [28] can be extended to solve the SFP remains an interesting 
open topic. For example, it is yet not clear whether the dual approach to (7) of [29] can be extended to the SFP. The original algorithm given in [15] involves the computation of the inverse $A^{-1}$ (assuming the existence of the inverse of $A$ ), and thus has not become popular. A seemingly more popular algorithm that solves the SFP is the CQ algorithm of Byrne $[16,21]$ which is found to be a gradient-projection method (GPM) in convex minimization. It is also a special case of the proximal forward-backward splitting method [30]. The CQ algorithm only involves the computation of the projections $P_{C}$ and $P_{Q}$ onto the sets $C$ and $Q$, respectively, and is therefore implementable in the case where $P_{C}$ and $P_{\mathrm{Q}}$ have closed-form expressions; for example, $C$ and $Q$ are closed balls or halfspaces. However, it remains a challenge how to implement the $C Q$ algorithm in the case where the projections $P_{C}$ and/or $P_{Q}$ fail to have closed-form expressions, though theoretically we can prove the (weak) convergence of the algorithm.

Very recently, $\mathrm{Xu}$ [20] gave a continuation of the study on the CQ algorithm and its convergence. He applied Mann's algorithm to the SFP and purposed an averaged CQ algorithm which was proved to be weakly convergent to a solution of the SFP. He also established the strong convergence result, which shows that the minimum-norm solution can be obtained.

Furthermore, Korpelevič [11] introduced the so-called extragradient method for finding a solution of a saddle point problem. He proved that the sequences generated by the proposed iterative algorithm converge to a solution of the saddle point problem.

Throughout this paper, assume that the SFP is consistent; that is, the solution set $\Gamma$ of the SFP is nonempty. Let $f$ : $\mathscr{H}_{1} \rightarrow \mathbf{R}$ be a continuous differentiable function. The minimization problem

$$
\min _{x \in C} f(x):=\frac{1}{2}\left\|A x-P_{\mathrm{Q}} A x\right\|^{2}
$$

is ill posed. Therefore, $\mathrm{Xu}$ [20] considered the following Tikhonov regularization problem:

$$
\min _{x \in C} f_{\alpha}(x):=\frac{1}{2}\left\|A x-P_{Q} A x\right\|^{2}+\frac{1}{2} \alpha\|x\|^{2},
$$

where $\alpha>0$ is the regularization parameter. The regularized minimization (9) has a unique solution which is denoted by $x_{\alpha}$. The following results are easy to prove.

Proposition 3 (see [31, Proposition 3.1]). Given $x^{*} \in \mathscr{H}_{1}$, the following statements are equivalent:

(i) $x^{*}$ solves the SFP;

(ii) $x^{*}$ solves the fixed point equation

$$
P_{C}(I-\lambda \nabla f) x^{*}=x^{*}
$$

where $\lambda>0, \nabla f=A^{*}\left(I-P_{\mathrm{Q}}\right) A$ and $A^{*}$ is the adjoint of $A$;

(iii) $x^{*}$ solves the variational inequality problem (VIP) of finding $x^{*} \in C$ such that

$$
\left\langle\nabla f\left(x^{*}\right), x-x^{*}\right\rangle \geq 0, \quad \forall x \in C .
$$

It is clear from Proposition 3 that

$$
\Gamma=\operatorname{Fix}\left(P_{C}(I-\lambda \nabla f)\right)=\operatorname{VI}(C, \nabla f)
$$

for all $\lambda>0$, where $\operatorname{Fix}\left(P_{C}(I-\lambda \nabla f)\right)$ and $\operatorname{VI}(C, \nabla f)$ denote the set of fixed points of $P_{C}(I-\lambda \nabla f)$ and the solution set of VIP (11), respectively.

Proposition 4 (see [31]). The following statements hold:

(i) the gradient

$$
\nabla f_{\alpha}=\nabla f+\alpha I=A^{*}\left(I-P_{\mathrm{Q}}\right) A+\alpha I
$$

is $\left(\alpha+\|A\|^{2}\right)$-Lipschitz continuous and $\alpha$-strongly monotone;

(ii) the mapping $P_{C}\left(I-\lambda \nabla f_{\alpha}\right)$ is a contraction with coefficient

$$
\sqrt{1-\lambda\left(2 \alpha-\lambda\left(\|A\|^{2}+\alpha\right)^{2}\right)}\left(\leq \sqrt{1-\alpha \lambda} \leq 1-\frac{1}{2} \alpha \lambda\right),
$$

where $0<\lambda \leq \alpha /\left(\|A\|^{2}+\alpha\right)^{2}$;

(iii) if the SFP is consistent, then the strong $\lim _{\alpha \rightarrow 0} x_{\alpha}$ exists and is the minimum-norm solution of the SFP.

Very recently, by combining the regularization method and extragradient method due to Nadezhkina and Takahashi [32], Ceng et al. [31] proposed an extragradient algorithm with regularization and proved that the sequences generated by the proposed algorithm converge weakly to an element of $\operatorname{Fix}(S) \cap \Gamma$, where $S: C \rightarrow C$ is a nonexpansive mapping.

Theorem 5 (see [31, Theorem 3.1]). Let $S: C \rightarrow C$ be a nonexpansive mapping such that $\operatorname{Fix}(S) \cap \Gamma \neq \emptyset$. Let $\left\{x_{n}\right\}$ and $\left\{y_{n}\right\}$ be the sequences in $C$ generated by the following extragradient algorithm:

$$
\begin{gathered}
x_{0}=x \in C \quad \text { chosen arbitrarily, } \\
y_{n}=P_{C}\left(x_{n}-\lambda_{n} \nabla f_{\alpha_{n}}\left(x_{n}\right)\right), \\
x_{n+1}=\beta_{n} x_{n}+\left(1-\beta_{n}\right) S P_{C}\left(x_{n}-\lambda_{n} \nabla f_{\alpha_{n}}\left(y_{n}\right)\right), \quad \forall n \geq 0,
\end{gathered}
$$

where $\sum_{n=0}^{\infty} \alpha_{n}<\infty,\left\{\lambda_{n}\right\} \subset[a, b]$ for some $a, b \in\left(0,1 /\|A\|^{2}\right)$ and $\left\{\beta_{n}\right\} \subset[c, d]$ for some $c, d \in(0,1)$. Then, both sequences $\left\{x_{n}\right\}$ and $\left\{y_{n}\right\}$ converge weakly to an element $\widehat{x} \in \operatorname{Fix}(S) \cap \Gamma$.

Motivated and inspired by the research going on this area, we propose and analyze the following Mann-type extragradient iterative algorithms with regularization for finding a common element of the solution set of the GSVI (3), the solution set of the SFP (6), and the fixed point set of a strictly pseudocontractive mapping $S: C \rightarrow C$.

Algorithm 6. Let $\mu_{i} \in\left(0,2 \beta_{i}\right)$ for $i=1,2,\left\{\alpha_{n}\right\} \subset(0, \infty)$, $\left\{\lambda_{n}\right\} \subset\left(0,1 /\|A\|^{2}\right)$ and $\left\{\sigma_{n}\right\},\left\{\tau_{n}\right\},\left\{\beta_{n}\right\},\left\{\gamma_{n}\right\},\left\{\delta_{n}\right\} \subset[0,1]$ such that $\sigma_{n}+\tau_{n} \leq 1$ and $\beta_{n}+\gamma_{n}+\delta_{n}=1$ for all $n \geq 0$. For 
given $x_{0} \in C$ arbitrarily, let $\left\{x_{n}\right\},\left\{y_{n}\right\},\left\{z_{n}\right\}$ be the sequences generated by the Mann-type extragradient iterative scheme with regularization

$$
\begin{gathered}
z_{n}=P_{C}\left(x_{n}-\lambda_{n} \nabla f_{\alpha_{n}}\left(x_{n}\right)\right), \\
y_{n}=\sigma_{n} x_{n}+\tau_{n} P_{C}\left(x_{n}-\lambda_{n} \nabla f_{\alpha_{n}}\left(z_{n}\right)\right) \\
+\left(1-\sigma_{n}-\tau_{n}\right) P_{C}\left[P_{C}\left(z_{n}-\mu_{2} B_{2} z_{n}\right)\right. \\
\left.-\mu_{1} B_{1} P_{C}\left(z_{n}-\mu_{2} B_{2} z_{n}\right)\right], \\
x_{n+1}=\beta_{n} x_{n}+\gamma_{n} y_{n}+\delta_{n} S y_{n}, \quad \forall n \geq 0 .
\end{gathered}
$$

Under appropriate assumptions, it is proven that all the sequences $\left\{x_{n}\right\},\left\{y_{n}\right\},\left\{z_{n}\right\}$ converge weakly to an element $\bar{x} \in$ $\operatorname{Fix}(S) \cap \Xi \cap \Gamma$. Furthermore, $(\bar{x}, \bar{y})$ is a solution of the GSVI (3), where $\bar{y}=P_{C}\left(\bar{x}-\mu_{2} B_{2} \bar{x}\right)$.

Algorithm 7. Let $\mu_{i} \in\left(0,2 \beta_{i}\right)$ for $i=1,2,\left\{\alpha_{n}\right\} \subset(0, \infty)$, $\left\{\lambda_{n}\right\} \subset\left(0,1 /\|A\|^{2}\right)$ and $\left\{\sigma_{n}\right\},\left\{\beta_{n}\right\},\left\{\gamma_{n}\right\},\left\{\delta_{n}\right\} \subset[0,1]$ such that $\beta_{n}+\gamma_{n}+\delta_{n}=1$ for all $n \geq 0$. For given $x_{0} \in C$ arbitrarily, let $\left\{x_{n}\right\},\left\{u_{n}\right\},\left\{\widetilde{u}_{n}\right\}$ be the sequences generated by the Mann-type extragradient iterative scheme with regularization

$$
\begin{gathered}
u_{n}=P_{C}\left[P_{C}\left(x_{n}-\mu_{2} B_{2} x_{n}\right)-\mu_{1} B_{1} P_{C}\left(x_{n}-\mu_{2} B_{2} x_{n}\right)\right], \\
\tilde{u}_{n}=P_{C}\left(u_{n}-\lambda_{n} \nabla f_{\alpha_{n}}\left(u_{n}\right)\right), \\
y_{n}=\sigma_{n} x_{n}+\left(1-\sigma_{n}\right) P_{C}\left(u_{n}-\lambda_{n} \nabla f_{\alpha_{n}}\left(\widetilde{u}_{n}\right)\right), \\
x_{n+1}=\beta_{n} x_{n}+\gamma_{n} y_{n}+\delta_{n} S y_{n}, \quad \forall n \geq 0 .
\end{gathered}
$$

Also, under mild conditions, it is shown that all the sequences $\left\{x_{n}\right\},\left\{u_{n}\right\},\left\{\widetilde{u}_{n}\right\}$ converge weakly to an element $\bar{x} \in$ $\operatorname{Fix}(S) \cap \Xi \cap \Gamma$. Furthermore, $(\bar{x}, \bar{y})$ is a solution of the GSVI (3), where $\bar{y}=P_{C}\left(\bar{x}-\mu_{2} B_{2} \bar{x}\right)$.

Observe that both [20, Theorem 5.7] and [31, Theorem 3.1] are weak convergence results for solving the SFP and so are our results as well. But our problem of finding an element of $\operatorname{Fix}(S) \cap \Xi \cap \Gamma$ is more general than the corresponding ones in [20, Theorem 5.7] and [31, Theorem 3.1], respectively. Hence, there is no doubt that our weak convergence results are very interesting and quite valuable. Because the Manntype extragradient iterative schemes (16) and (17) with regularization involve two inverse strongly monotone mappings $B_{1}$ and $B_{2}$, a $k$-strictly pseudocontractive self-mapping $S$ and several parameter sequences, they are more flexible and more subtle than the corresponding ones in [20, Theorem 5.7] and [31, Theorem 3.1], respectively. Furthermore, the hybrid extragradient iterative scheme (5) is extended to develop the Mann-type extragradient iterative schemes (16) and (17) with regularization. In our results, the Mann-type extragradient iterative schemes (16) and (17) with regularization lack the requirement of boundedness for the domain in which various mappings are defined; see, for example, Yao et al. [7, Theorem 3.2]. Therefore, our results represent the modification, supplementation, extension, and improvement of [20, Theorem 5.7], [31, Theorem 3.1], [14, Theorem 3.1], and [7, Theorem $3.2]$.

\section{Preliminaries}

Let $\mathscr{H}$ be a real Hilbert space, whose inner product and norm are denoted by $\langle\cdot, \cdot\rangle$ and $\|\cdot\|$, respectively. Let $K$ be a nonempty, closed, and convex subset of $\mathscr{H}$. Now we present some known definitions and results which will be used in the sequel.

The metric (or nearest point) projection from $\mathscr{H}$ onto $K$ is the mapping $P_{K}: \mathscr{H} \rightarrow K$ which assigns to each point $x \in \mathscr{H}$ the unique point $P_{K} x \in K$ satisfying the property

$$
\left\|x-P_{K} x\right\|=\inf _{y \in K}\|x-y\|=: d(x, K) .
$$

Some important properties of projections are gathered in the following proposition.

Proposition 8. For given $x \in \mathscr{H}$ and $z \in K$ :

(i) $z=P_{K} x \Leftrightarrow\langle x-z, y-z\rangle \leq 0$, for all $y \in K$;

(ii) $z=P_{K} x \Leftrightarrow\|x-z\|^{2} \leq\|x-y\|^{2}-\|y-z\|^{2}$, for all $y \in K$

(iii) $\left\langle P_{K} x-P_{K} y, x-y\right\rangle \geq\left\|P_{K} x-P_{K} y\right\|^{2}$, for all $y \in$ $\mathscr{H}$, which hence implies that $P_{K}$ is nonexpansive and monotone.

Definition 9. A mapping $T: \mathscr{H} \rightarrow \mathscr{H}$ is said to be

(a) nonexpansive if

$$
\|T x-T y\| \leq\|x-y\|, \quad \forall x, y \in \mathscr{H}
$$

(b) firmly nonexpansive if $2 T-I$ is nonexpansive, or equivalently,

$$
\langle x-y, T x-T y\rangle \geq\|T x-T y\|^{2}, \quad \forall x, y \in \mathscr{H} ;
$$

alternatively, $T$ is firmly nonexpansive if and only if $T$ can be expressed as

$$
T=\frac{1}{2}(I+S),
$$

where $S: \mathscr{H} \rightarrow \mathscr{H}$ is nonexpansive; projections are firmly nonexpansive.

Definition 10. Let $T$ be a nonlinear operator with domain $D(T) \subseteq \mathscr{H}$ and range $R(T) \subseteq \mathscr{H}$.

(a) $T$ is said to be monotone if

$$
\langle x-y, T x-T y\rangle \geq 0, \quad \forall x, y \in D(T) .
$$

(b) Given a number $\beta>0, T$ is said to be $\beta$-strongly monotone if

$$
\langle x-y, T x-T y\rangle \geq \beta\|x-y\|^{2}, \quad \forall x, y \in D(T) .
$$

(c) Given a number $v>0, T$ is said to be $v$-inverse strongly monotone $(\nu$-ism) if

$\langle x-y, T x-T y\rangle \geq v\|T x-T y\|^{2}, \quad \forall x, y \in D(T)$. 
It can be easily seen that if $S$ is nonexpansive, then $I-S$ is monotone. It is also easy to see that a projection $P_{K}$ is 1-ism.

Inverse strongly monotone (also referred to as cocoercive) operators have been applied widely in solving practical problems in various fields, for instance, in traffic assignment problems; see, for example, $[33,34]$.

Definition 11. A mapping $T: \mathscr{H} \rightarrow \mathscr{H}$ is said to be an averaged mapping if it can be written as the average of the identity $I$ and a nonexpansive mapping, that is,

$$
T \equiv(1-\alpha) I+\alpha S
$$

where $\alpha \in(0,1)$ and $S: \mathscr{H} \rightarrow \mathscr{H}$ is nonexpansive. More precisely, when the last equality holds, we say that $T$ is $\alpha$ averaged. Thus, firmly nonexpansive mappings (in particular, projections) are 1/2-averaged maps.

Proposition 12 (see [21]). Let $T: \mathscr{H} \rightarrow \mathscr{H}$ be a given mapping.

(i) $T$ is nonexpansive if and only if the complement $I-T$ is $1 / 2$-ism.

(ii) If $T$ is $\nu$-ism, then for $\gamma>0, \gamma T$ is $\nu / \gamma$-ism.

(iii) $T$ is averaged if and only if the complement $I-T$ is $\nu$-ism for some $v>1 / 2$. Indeed, for $\alpha \in(0,1), T$ is $\alpha$-averaged if and only if $I-T$ is $1 / 2 \alpha$-ism.

Proposition 13 (see $[21,35])$. Let $S, T, V: \mathscr{H} \rightarrow \mathscr{H}$ be given operators.

(i) If $T=(1-\alpha) S+\alpha V$ for some $\alpha \in(0,1)$ and if $S$ is averaged and $V$ is nonexpansive, then $T$ is averaged.

(ii) $T$ is firmly nonexpansive if and only if the complement $I-T$ is firmly nonexpansive.

(iii) If $T=(1-\alpha) S+\alpha V$ for some $\alpha \in(0,1)$ and if $S$ is firmly nonexpansive and $V$ is nonexpansive, then $T$ is averaged.

(iv) The composite of finitely many averaged mappings is averaged. That is, if each of the mappings $\left\{T_{i}\right\}_{i=1}^{N}$ is averaged, then so is the composite $T_{1} \circ T_{2} \circ \cdots \circ T_{N}$. In particular, if $T_{1}$ is $\alpha_{1}$-averaged and $T_{2}$ is $\alpha_{2}$-averaged, where $\alpha_{1}, \alpha_{2} \in(0,1)$, then the composite $T_{1} \circ T_{2}$ is $\alpha$ averaged, where $\alpha=\alpha_{1}+\alpha_{2}-\alpha_{1} \alpha_{2}$.

(v) If the mappings $\left\{T_{i}\right\}_{i=1}^{N}$ are averaged and have a common fixed point, then

$$
\bigcap_{i=1}^{N} \operatorname{Fix}\left(T_{i}\right)=\operatorname{Fix}\left(T_{1} \cdots T_{N}\right) .
$$

The notation $\operatorname{Fix}(T)$ denotes the set of all fixed points of the mapping $T$, that is, $\operatorname{Fix}(T)=\{x \in \mathscr{H}: T x=x\}$.
It is clear that in a real Hilbert space $\mathscr{H}, S: C \rightarrow C$ is $k$-strictly pseudocontractive if and only if there holds the following inequality:

$$
\begin{array}{r}
\langle S x-S y, x-y\rangle \\
\leq\|x-y\|^{2}-\frac{1-k}{2}\|(I-S) x-(I-S) y\|^{2}, \\
\forall x, y \in C .
\end{array}
$$

This immediately implies that if $S$ is a $k$-strictly pseudocontractive mapping, then $I-S$ is $(1-k) / 2$-inverse strongly monotone; for further detail, we refer to [9] and the references therein. It is well known that the class of strict pseudocontractions strictly includes the class of nonexpansive mappings.

The following elementary result in the real Hilbert spaces is quite well known.

Lemma 14 (see [36]). Let $\mathscr{H}$ be a real Hilbert space. Then, for all $x, y \in \mathscr{H}$ and $\lambda \in[0,1]$,

$$
\begin{aligned}
\|\lambda x+(1-\lambda) y\|^{2}= & \lambda\|x\|^{2}+(1-\lambda)\|y\|^{2} \\
& -\lambda(1-\lambda)\|x-y\|^{2} .
\end{aligned}
$$

Lemma 15 (see [37, Proposition 2.1]). Let $C$ be a nonempty closed convex subset of a real Hilbert space $\mathscr{H}$ and $S: C \rightarrow C$ be a mapping.

(i) If $S$ is a $k$-strict pseudocontractive mapping, then $S$ satisfies the Lipschitz condition

$$
\|S x-S y\| \leq \frac{1+k}{1-k}\|x-y\|, \quad \forall x, y \in C .
$$

(ii) If $S$ is a $k$-strict pseudocontractive mapping, then the mapping $I-S$ is semiclosed at 0 , that is, if $\left\{x_{n}\right\}$ is a sequence in $C$ such that $x_{n} \rightarrow \tilde{x}$ weakly and (I S) $x_{n} \rightarrow 0$ strongly, then $(I-S) \tilde{x}=0$.

(iii) If $S$ is $k$-(quasi-)strict pseudocontraction, then the fixed point set $\operatorname{Fix}(S)$ of $S$ is closed and convex so that the projection $P_{\mathrm{Fix}(S)}$ is well defined.

The following lemma plays a key role in proving weak convergence of the sequences generated by our algorithms.

Lemma 16 (see [38, p. 80]). Let $\left\{a_{n}\right\}_{n=0}^{\infty},\left\{b_{n}\right\}_{n=0}^{\infty}$, and $\left\{\delta_{n}\right\}_{n=0}^{\infty}$ be sequences of nonnegative real numbers satisfying the inequality

$$
a_{n+1} \leq\left(1+\delta_{n}\right) a_{n}+b_{n}, \quad \forall n \geq 0 .
$$

If $\sum_{n=0}^{\infty} \delta_{n}<\infty$ and $\sum_{n=0}^{\infty} b_{n}<\infty$, then $\lim _{n \rightarrow \infty} a_{n}$ exists. If, in addition, $\left\{a_{n}\right\}_{n=0}^{\infty}$ has a subsequence which converges to zero, then $\lim _{n \rightarrow \infty} a_{n}=0$.

Corollary 17 (see [39, p. 303]). Let $\left\{a_{n}\right\}_{n=0}^{\infty}$ and $\left\{b_{n}\right\}_{n=0}^{\infty}$ be two sequences of nonnegative real numbers satisfying the inequality

$$
a_{n+1} \leq a_{n}+b_{n}, \quad \forall n \geq 0 .
$$

If $\sum_{n=0}^{\infty} b_{n}$ converges, then $\lim _{n \rightarrow \infty} a_{n}$ exists. 
Lemma 18 (see [7]). Let $C$ be a nonempty closed convex subset of a real Hilbert space $\mathscr{H}$. Let $S: C \rightarrow C$ be a $k$-strictly pseudocontractive mapping. Let $\gamma$ and $\delta$ be two nonnegative real numbers such that $(\gamma+\delta) k \leq \gamma$. Then

$$
\|\gamma(x-y)+\delta(S x-S y)\| \leq(\gamma+\delta)\|x-y\|, \quad \forall x, y \in C .
$$

The following lemma is an immediate consequence of an inner product.

Lemma 19. In a real Hilbert space $\mathscr{H}$, there holds the inequality

$$
\|x+y\|^{2} \leq\|x\|^{2}+2\langle y, x+y\rangle, \quad \forall x, y \in \mathscr{H} .
$$

Let $K$ be a nonempty closed convex subset of a real Hilbert space $\mathscr{H}$ and let $F: K \rightarrow \mathscr{H}$ be a monotone mapping. The variational inequality problem (VIP) is to find $x \in K$ such that

$$
\langle F x, y-x\rangle \geq 0, \quad \forall y \in K
$$

The solution set of the VIP is denoted by $\operatorname{VI}(K, F)$. It is well known that

$$
x \in \mathrm{VI}(K, F) \Longleftrightarrow x=P_{K}(x-\lambda F x), \quad \forall \lambda>0 .
$$

A set-valued mapping $T: \mathscr{H} \rightarrow 2^{\mathscr{H}}$ is called monotone if for all $x, y \in \mathscr{H}, f \in T x$ and $g \in T y$ imply that $\langle x-y, f-$ $g\rangle \geq 0$. A monotone set-valued mapping $T: \mathscr{H} \rightarrow 2^{\mathscr{H}}$ is called maximal if its graph $\mathrm{Gph}(T)$ is not properly contained in the graph of any other monotone set-valued mapping. It is known that a monotone set-valued mapping $T: \mathscr{H} \rightarrow 2^{\mathscr{H}}$ is maximal if and only if for $(x, f) \in \mathscr{H} \times \mathscr{H},\langle x-y, f-g\rangle \geq 0$ for every $(y, g) \in \mathrm{Gph}(T)$ implies that $f \in T x$. Let $F: K \rightarrow$ $\mathscr{H}$ be a monotone and Lipschitz continuous mapping and let $N_{K} v$ be the normal cone to $K$ at $v \in K$, that is,

$$
N_{K} v=\{w \in \mathscr{H}:\langle v-u, w\rangle \geq 0, \forall u \in K\} .
$$

Define

$$
T v= \begin{cases}F v+N_{K} v, & \text { if } v \in K \\ \emptyset, & \text { if } v \notin K\end{cases}
$$

It is known that in this case the mapping $T$ is maximal monotone, and $0 \in T v$ if and only if $v \in \operatorname{VI}(K, F)$; for further details, we refer to [40] and the references therein.

\section{Main Results}

In this section, we first prove the weak convergence of the sequences generated by the Mann-type extragradient iterative algorithm (16) with regularization.

Theorem 20. Let $C$ be a nonempty closed convex subset of a real Hilbert space $\mathscr{H}_{1}$. Let $A \in B\left(\mathscr{H}_{1}, \mathscr{H}_{2}\right)$ and $B_{i}: C \rightarrow \mathscr{H}_{1}$ be $\beta_{i}$-inverse strongly monotone for $i=1,2$. Let $S: C \rightarrow C$ be a $k$-strictly pseudocontractive mapping such that $\operatorname{Fix}(S) \cap$
$\Xi \cap \Gamma \neq \emptyset$. For given $x_{0} \in C$ arbitrarily, let $\left\{x_{n}\right\},\left\{y_{n}\right\},\left\{z_{n}\right\}$ be the sequences generated by the Mann-type extragradient iterative algorithm (16) with regularization, where $\mu_{i} \in\left(0,2 \beta_{i}\right)$ for $i=$ $1,2,\left\{\alpha_{n}\right\} \subset(0, \infty),\left\{\lambda_{n}\right\} \subset\left(0,1 /\|A\|^{2}\right)$ and $\left\{\sigma_{n}\right\},\left\{\tau_{n}\right\},\left\{\beta_{n}\right\}$, $\left\{\gamma_{n}\right\},\left\{\delta_{n}\right\} \subset[0,1]$ such that

(i) $\sum_{n=0}^{\infty} \alpha_{n}<\infty$;

(ii) $\beta_{n}+\gamma_{n}+\delta_{n}=1$ and $\left(\gamma_{n}+\delta_{n}\right) k \leq \gamma_{n}$ for all $n \geq 0$;

(iii) $\sigma_{n}+\tau_{n} \leq 1$ for all $n \geq 0$;

(iv) $0<\liminf _{n \rightarrow \infty} \tau_{n} \leq \lim \sup _{n \rightarrow \infty}\left(\sigma_{n}+\tau_{n}\right)<1$;

(v) $0<\liminf _{n \rightarrow \infty} \beta_{n} \leq \limsup _{n \rightarrow \infty} \beta_{n}<1$ and $\liminf _{n \rightarrow \infty} \delta_{n}>0$;

(vi) $0<\liminf _{n \rightarrow \infty} \lambda_{n} \leq \lim \sup _{n \rightarrow \infty} \lambda_{n}<1 /\|A\|^{2}$.

Then all the sequences $\left\{x_{n}\right\},\left\{y_{n}\right\},\left\{z_{n}\right\}$ converge weakly to an element $\bar{x} \in \operatorname{Fix}(S) \cap \Xi \cap \Gamma$. Furthermore, $(\bar{x}, \bar{y})$ is a solution of GSVI (3), where $\bar{y}=P_{C}\left(\bar{x}-\mu_{2} B_{2} \bar{x}\right)$.

Proof. First, taking into account $0<\liminf _{n \rightarrow \infty} \lambda_{n} \leq$ $\limsup _{n \rightarrow \infty} \lambda_{n}<1 /\|A\|^{2}$, without loss of generality we may assume that $\left\{\lambda_{n}\right\} \subset[a, b]$ for some $a, b \in\left(0,1 /\|A\|^{2}\right)$.

Now, let us show that $P_{C}\left(I-\lambda \nabla f_{\alpha}\right)$ is $\zeta$-averaged for each $\lambda \in\left(0,2 /\left(\alpha+\|A\|^{2}\right)\right)$, where

$$
\zeta=\frac{2+\lambda\left(\alpha+\|A\|^{2}\right)}{4} \in(0,1) \text {. }
$$

Indeed, it is easy to see that $\nabla f=A^{*}\left(I-P_{\mathrm{Q}}\right) A$ is $1 /\|A\|^{2}$ ism, that is,

$$
\langle\nabla f(x)-\nabla f(y), x-y\rangle \geq \frac{1}{\|A\|^{2}}\|\nabla f(x)-\nabla f(y)\|^{2} .
$$

Observe that

$$
\begin{aligned}
\left(\alpha+\|A\|^{2}\right)\left\langle\nabla f_{\alpha}(x)-\nabla f_{\alpha}(y), x-y\right\rangle & \\
= & \left(\alpha+\|A\|^{2}\right)\left[\alpha\|x-y\|^{2}\right. \\
& \quad+\langle\nabla f(x)-\nabla f(y), x-y\rangle] \\
= & \alpha^{2}\|x-y\|^{2}+\alpha\langle\nabla f(x)-\nabla f(y), x-y\rangle \\
& +\alpha\|A\|^{2}\|x-y\|^{2} \\
& +\|A\|^{2}\langle\nabla f(x)-\nabla f(y), x-y\rangle \\
\geq & \alpha^{2}\|x-y\|^{2}+2 \alpha\langle\nabla f(x)-\nabla f(y), x-y\rangle \\
& +\|\nabla f(x)-\nabla f(y)\|^{2} \\
= & \|\alpha(x-y)+\nabla f(x)-\nabla f(y)\|^{2} \\
= & \left\|\nabla f_{\alpha}(x)-\nabla f_{\alpha}(y)\right\|^{2} .
\end{aligned}
$$

Hence, it follows that $\nabla f_{\alpha}=\alpha I+A^{*}\left(I-P_{\mathrm{Q}}\right) A$ is $1 /\left(\alpha+\|A\|^{2}\right)$-ism. Thus, $\lambda \nabla f_{\alpha}$ is $1 / \lambda\left(\alpha+\|A\|^{2}\right)$-ism according to Proposition 12(ii). By Proposition 12(iii), the complement 
$I-\lambda \nabla f_{\alpha}$ is $\lambda\left(\alpha+\|A\|^{2}\right) / 2$-averaged. Therefore, noting that $P_{C}$ is $1 / 2$-averaged and utilizing Proposition 13(iv), we know that for each $\lambda \in\left(0,2 /\left(\alpha+\|A\|^{2}\right)\right), P_{C}\left(I-\lambda \nabla f_{\alpha}\right)$ is $\zeta$-averaged with

$$
\begin{aligned}
\zeta & =\frac{1}{2}+\frac{\lambda\left(\alpha+\|A\|^{2}\right)}{2}-\frac{1}{2} \cdot \frac{\lambda\left(\alpha+\|A\|^{2}\right)}{2} \\
& =\frac{2+\lambda\left(\alpha+\|A\|^{2}\right)}{4} \in(0,1) .
\end{aligned}
$$

This shows that $P_{C}\left(I-\lambda \nabla f_{\alpha}\right)$ is nonexpansive. Furthermore, for $\left\{\lambda_{n}\right\} \subset[a, b]$ with $a, b \in\left(0,1 /\|A\|^{2}\right)$, we have

$$
a \leq \inf _{n \geq 0} \lambda_{n} \leq \sup _{n \geq 0} \lambda_{n} \leq b<\frac{1}{\|A\|^{2}}=\lim _{n \rightarrow \infty} \frac{1}{\alpha_{n}+\|A\|^{2}} .
$$

Without loss of generality, we may assume that

$$
a \leq \inf _{n \geq 0} \lambda_{n} \leq \sup _{n \geq 0} \lambda_{n} \leq b<\frac{1}{\alpha_{n}+\|A\|^{2}}, \quad \forall n \geq 0 .
$$

Consequently, it follows that for each integer $n \geq 0, P_{C}(I-$ $\left.\lambda_{n} \nabla f_{\alpha_{n}}\right)$ is $\zeta_{n}$-averaged with

$$
\begin{aligned}
\zeta_{n} & =\frac{1}{2}+\frac{\lambda_{n}\left(\alpha_{n}+\|A\|^{2}\right)}{2}-\frac{1}{2} \cdot \frac{\lambda_{n}\left(\alpha_{n}+\|A\|^{2}\right)}{2} \\
& =\frac{2+\lambda_{n}\left(\alpha_{n}+\|A\|^{2}\right)}{4} \in(0,1) .
\end{aligned}
$$

This immediately implies that $P_{C}\left(I-\lambda_{n} \nabla f_{\alpha_{n}}\right)$ is nonexpansive for all $n \geq 0$.

Next we divide the remainder of the proof into several steps.

Step 1. $\left\{x_{n}\right\}$ is bounded.

Indeed, take $p \in \operatorname{Fix}(S) \cap \Xi \cap \Gamma$ arbitrarily. Then $S p=p$, $P_{C}(I-\lambda \nabla f) p=p$ for $\lambda \in\left(0,2 /\|A\|^{2}\right)$, and

$$
p=P_{C}\left[P_{C}\left(p-\mu_{2} B_{2} p\right)-\mu_{1} B_{1} P_{C}\left(p-\mu_{2} B_{2} p\right)\right] .
$$

From (16), it follows that

$$
\begin{aligned}
\| z_{n}- & p \| \\
= & \left\|P_{C}\left(I-\lambda_{n} \nabla f_{\alpha_{n}}\right) x_{n}-P_{C}\left(I-\lambda_{n} \nabla f\right) p\right\| \\
\leq & \left\|P_{C}\left(I-\lambda_{n} \nabla f_{\alpha_{n}}\right) x_{n}-P_{C}\left(I-\lambda_{n} \nabla f_{\alpha_{n}}\right) p\right\| \\
& +\left\|P_{C}\left(I-\lambda_{n} \nabla f_{\alpha_{n}}\right) p-P_{C}\left(I-\lambda_{n} \nabla f\right) p\right\| \\
& \leq\left\|x_{n}-p\right\|+\left\|\left(I-\lambda_{n} \nabla f_{\alpha_{n}}\right) p-\left(I-\lambda_{n} \nabla f\right) p\right\| \\
\leq & \left\|x_{n}-p\right\|+\lambda_{n} \alpha_{n}\|p\| .
\end{aligned}
$$

Utilizing Lemma 19, we also have

$$
\begin{aligned}
\| z_{n}- & p \|^{2} \\
= & \left\|P_{C}\left(I-\lambda_{n} \nabla f_{\alpha_{n}}\right) x_{n}-P_{C}\left(I-\lambda_{n} \nabla f\right) p\right\|^{2} \\
= & \| P_{C}\left(I-\lambda_{n} \nabla f_{\alpha_{n}}\right) x_{n}-P_{C}\left(I-\lambda_{n} \nabla f_{\alpha_{n}}\right) p \\
& +P_{C}\left(I-\lambda_{n} \nabla f_{\alpha_{n}}\right) p-P_{C}\left(I-\lambda_{n} \nabla f\right) p \|^{2} \\
\leq & \left\|P_{C}\left(I-\lambda_{n} \nabla f_{\alpha_{n}}\right) x_{n}-P_{C}\left(I-\lambda_{n} \nabla f_{\alpha_{n}}\right) p\right\|^{2} \\
& +2\left\langle P_{C}\left(I-\lambda_{n} \nabla f_{\alpha_{n}}\right) p\right. \\
& \left.\quad-P_{C}\left(I-\lambda_{n} \nabla f\right) p, z_{n}-p\right\rangle \\
\leq & \left\|x_{n}-p\right\|^{2} \\
& +2\left\|P_{C}\left(I-\lambda_{n} \nabla f_{\alpha_{n}}\right) p-P_{C}\left(I-\lambda_{n} \nabla f\right) p\right\| \\
& \times\left\|z_{n}-p\right\| \\
\leq & \left\|x_{n}-p\right\|^{2} \\
& +2\left\|\left(I-\lambda_{n} \nabla f_{\alpha_{n}}\right) p-\left(I-\lambda_{n} \nabla f\right) p\right\|\left\|z_{n}-p\right\| \\
= & \left\|x_{n}-p\right\|^{2}+2 \lambda_{n} \alpha_{n}\|p\|\left\|z_{n}-p\right\| .
\end{aligned}
$$

For simplicity, we write $q=P_{C}\left(p-\mu_{2} B_{2} p\right), \widetilde{z}_{n}=P_{C}\left(z_{n}-\right.$ $\left.\mu_{2} B_{2} z_{n}\right)$,

$$
\begin{gathered}
u_{n}=P_{C}\left[P_{C}\left(z_{n}-\mu_{2} B_{2} z_{n}\right)-\mu_{1} B_{1} P_{C}\left(z_{n}-\mu_{2} B_{2} z_{n}\right)\right], \\
\bar{u}_{n}=P_{C}\left(x_{n}-\lambda_{n} \nabla f_{\alpha_{n}}\left(z_{n}\right)\right)
\end{gathered}
$$

for each $n \geq 0$. Then $y_{n}=\sigma_{n} x_{n}+\tau_{n} \bar{u}_{n}+\left(1-\sigma_{n}-\tau_{n}\right) u_{n}$ for each $n \geq 0$. Since $B_{i}: C \rightarrow \mathscr{H}_{1}$ is $\beta_{i}$-inverse strongly monotone and $0<\mu_{i}<2 \beta_{i}$ for $i=1,2$, we know that for all $n \geq 0$,

$$
\begin{aligned}
& \left\|u_{n}-p\right\|^{2} \\
& =\| P_{C}\left[P_{C}\left(z_{n}-\mu_{2} B_{2} z_{n}\right)\right. \\
& \left.\quad-\mu_{1} B_{1} P_{C}\left(z_{n}-\mu_{2} B_{2} z_{n}\right)\right]-p \|^{2} \\
& =\| P_{C}\left[P_{C}\left(z_{n}-\mu_{2} B_{2} z_{n}\right)-\mu_{1} B_{1} P_{C}\left(z_{n}-\mu_{2} B_{2} z_{n}\right)\right] \\
& \quad-P_{C}\left[P_{C}\left(p-\mu_{2} B_{2} p\right)-\mu_{1} B_{1} P_{C}\left(p-\mu_{2} B_{2} p\right)\right] \|^{2} \\
& \leq \|\left[P_{C}\left(z_{n}-\mu_{2} B_{2} z_{n}\right)-\mu_{1} B_{1} P_{C}\left(z_{n}-\mu_{2} B_{2} z_{n}\right)\right] \\
& \quad-\left[P_{C}\left(p-\mu_{2} B_{2} p\right)-\mu_{1} B_{1} P_{C}\left(p-\mu_{2} B_{2} p\right)\right] \|^{2} \\
& =\|\left[P_{C}\left(z_{n}-\mu_{2} B_{2} z_{n}\right)-P_{C}\left(p-\mu_{2} B_{2} p\right)\right] \\
& \quad-\mu_{1}\left[B_{1} P_{C}\left(z_{n}-\mu_{2} B_{2} z_{n}\right)-B_{1} P_{C}\left(p-\mu_{2} B_{2} p\right)\right] \|^{2}
\end{aligned}
$$




$$
\begin{aligned}
\leq & \left\|P_{C}\left(z_{n}-\mu_{2} B_{2} z_{n}\right)-P_{C}\left(p-\mu_{2} B_{2} p\right)\right\|^{2} \\
& -\mu_{1}\left(2 \beta_{1}-\mu_{1}\right) \| B_{1} P_{C}\left(z_{n}-\mu_{2} B_{2} z_{n}\right) \\
& -B_{1} P_{C}\left(p-\mu_{2} B_{2} p\right) \|^{2} \\
\leq & \left\|\left(z_{n}-\mu_{2} B_{2} z_{n}\right)-\left(p-\mu_{2} B_{2} p\right)\right\|^{2} \\
& -\mu_{1}\left(2 \beta_{1}-\mu_{1}\right)\left\|B_{1} \widetilde{z}_{n}-B_{1} q\right\|^{2} \\
= & \left\|\left(z_{n}-p\right)-\mu_{2}\left(B_{2} z_{n}-B_{2} p\right)\right\|^{2} \\
& -\mu_{1}\left(2 \beta_{1}-\mu_{1}\right)\left\|B_{1} \widetilde{z}_{n}-B_{1} q\right\|^{2} \\
\leq & \left\|z_{n}-p\right\|^{2}-\mu_{2}\left(2 \beta_{2}-\mu_{2}\right)\left\|B_{2} z_{n}-B_{2} p\right\|^{2} \\
& -\mu_{1}\left(2 \beta_{1}-\mu_{1}\right)\left\|B_{1} \tilde{z}_{n}-B_{1} q\right\|^{2} \leq\left\|z_{n}-p\right\|^{2} .
\end{aligned}
$$

Furthermore, by Proposition 8(ii), we have

$$
\begin{aligned}
\| \bar{u}_{n}- & p \|^{2} \\
\leq & \left\|x_{n}-\lambda_{n} \nabla f_{\alpha_{n}}\left(z_{n}\right)-p\right\|^{2}-\left\|x_{n}-\lambda_{n} \nabla f_{\alpha_{n}}\left(z_{n}\right)-\bar{u}_{n}\right\|^{2} \\
= & \left\|x_{n}-p\right\|^{2}-\left\|x_{n}-\bar{u}_{n}\right\|^{2}+2 \lambda_{n}\left\langle\nabla f_{\alpha_{n}}\left(z_{n}\right), p-\bar{u}_{n}\right\rangle \\
= & \left\|x_{n}-p\right\|^{2}-\left\|x_{n}-\bar{u}_{n}\right\|^{2} \\
& +2 \lambda_{n}\left(\left\langle\nabla f_{\alpha_{n}}\left(z_{n}\right)-\nabla f_{\alpha_{n}}(p), p-z_{n}\right\rangle\right. \\
& \quad \quad\left\langle\nabla f_{\alpha_{n}}(p), p-z_{n}\right\rangle \\
& \quad\left\langle\left\langle\nabla f_{\alpha_{n}}\left(z_{n}\right), z_{n}-\bar{u}_{n}\right\rangle\right) \\
\leq & \left\|x_{n}-p\right\|^{2}-\left\|x_{n}-\bar{u}_{n}\right\|^{2} \\
& +2 \lambda_{n}\left(\left\langle\nabla f_{\alpha_{n}}(p), p-z_{n}\right\rangle+\left\langle\nabla f_{\alpha_{n}}\left(z_{n}\right), z_{n}-\bar{u}_{n}\right\rangle\right) \\
= & \left\|x_{n}-p\right\|^{2}-\left\|x_{n}-\bar{u}_{n}\right\|^{2} \\
& +2 \lambda_{n}\left[\left\langle\left(\alpha_{n} I+\nabla f\right) p, p-z_{n}\right\rangle+\left\langle\nabla f_{\alpha_{n}}\left(z_{n}\right), z_{n}-\bar{u}_{n}\right\rangle\right] \\
\leq & \left\|x_{n}-p\right\|^{2}-\left\|x_{n}-\bar{u}_{n}\right\|^{2} \\
& +2 \lambda_{n}\left[\alpha_{n}\left\langle p, p-z_{n}\right\rangle+\left\langle\nabla f_{\alpha_{n}}\left(z_{n}\right), z_{n}-\bar{u}_{n}\right\rangle\right] \\
= & \left\|x_{n}-p\right\|^{2}-\left\|x_{n}-z_{n}\right\|^{2} \\
& -2\left\langle x_{n}-z_{n}, z_{n}-\bar{u}_{n}\right\rangle-\left\|z_{n}-\bar{u}_{n}\right\|^{2} \\
& +2 \lambda_{n}\left[\alpha_{n}\left\langle p, p-z_{n}\right\rangle+\left\langle\nabla f_{\alpha_{n}}\left(z_{n}\right), z_{n}-\bar{u}_{n}\right\rangle\right] \\
& \left\|x_{n}-p\right\|^{2}-\left\|x_{n}-z_{n}\right\|^{2}-\left\|z_{n}-\bar{u}_{n}\right\|^{2} \\
& +2\left\langle x_{n}-\lambda_{n} \nabla f_{\alpha_{n}}\left(z_{n}\right)-z_{n}, \bar{u}_{n}-z_{n}\right\rangle \\
& +2 \lambda_{n} \alpha_{n}\left\langle p, p-z_{n}\right\rangle . \\
& \\
&
\end{aligned}
$$

Further, by Proposition 8(i), we have

$$
\begin{aligned}
\left\langle x_{n}-\right. & \left.\lambda_{n} \nabla f_{\alpha_{n}}\left(z_{n}\right)-z_{n}, \bar{u}_{n}-z_{n}\right\rangle \\
= & \left\langle x_{n}-\lambda_{n} \nabla f_{\alpha_{n}}\left(x_{n}\right)-z_{n}, \bar{u}_{n}-z_{n}\right\rangle \\
& +\left\langle\lambda_{n} \nabla f_{\alpha_{n}}\left(x_{n}\right)-\lambda_{n} \nabla f_{\alpha_{n}}\left(z_{n}\right), \bar{u}_{n}-z_{n}\right\rangle \\
\leq & \left\langle\lambda_{n} \nabla f_{\alpha_{n}}\left(x_{n}\right)-\lambda_{n} \nabla f_{\alpha_{n}}\left(z_{n}\right), \bar{u}_{n}-z_{n}\right\rangle \\
\leq & \lambda_{n}\left\|\nabla f_{\alpha_{n}}\left(x_{n}\right)-\nabla f_{\alpha_{n}}\left(z_{n}\right)\right\|\left\|\bar{u}_{n}-z_{n}\right\| \\
\leq & \lambda_{n}\left(\alpha_{n}+\|A\|^{2}\right)\left\|x_{n}-z_{n}\right\|\left\|\bar{u}_{n}-z_{n}\right\| .
\end{aligned}
$$

So, from (46), we obtain

$$
\begin{aligned}
\left\|\bar{u}_{n}-p\right\|^{2} \leq & \left\|x_{n}-p\right\|^{2}-\left\|x_{n}-z_{n}\right\|^{2}-\left\|z_{n}-\bar{u}_{n}\right\|^{2} \\
& +2\left\langle x_{n}-\lambda_{n} \nabla f_{\alpha_{n}}\left(z_{n}\right)-z_{n}, \bar{u}_{n}-z_{n}\right\rangle \\
& +2 \lambda_{n} \alpha_{n}\left\langle p, p-z_{n}\right\rangle \\
\leq & \left\|x_{n}-p\right\|^{2}-\left\|x_{n}-z_{n}\right\|^{2}-\left\|z_{n}-\bar{u}_{n}\right\|^{2} \\
& +2 \lambda_{n}\left(\alpha_{n}+\|A\|^{2}\right)\left\|x_{n}-z_{n}\right\|\left\|\bar{u}_{n}-z_{n}\right\| \\
& +2 \lambda_{n} \alpha_{n}\|p\|\left\|z_{n}-p\right\| \\
\leq & \left\|x_{n}-p\right\|^{2}-\left\|x_{n}-z_{n}\right\|^{2}-\left\|z_{n}-\bar{u}_{n}\right\|^{2} \\
& +\lambda_{n}^{2}\left(\alpha_{n}+\|A\|^{2}\right)^{2}\left\|x_{n}-z_{n}\right\|^{2} \\
& +\left\|z_{n}-\bar{u}_{n}\right\|^{2}+2 \lambda_{n} \alpha_{n}\|p\|\left\|z_{n}-p\right\| \\
= & \left\|x_{n}-p\right\|^{2}+2 \lambda_{n} \alpha_{n}\|p\|\left\|z_{n}-p\right\| \\
& +\left(\lambda_{n}^{2}\left(\alpha_{n}+\|A\|^{2}\right)^{2}-1\right)\left\|x_{n}-z_{n}\right\|^{2} \\
\leq & \left\|x_{n}-p\right\|^{2}+2 \lambda_{n} \alpha_{n}\|p\|\left\|z_{n}-p\right\| \\
\leq & \left\|x_{n}-p\right\|^{2}+2 \lambda_{n} \alpha_{n}\|p\|\left[\left\|x_{n}-p\right\|+\lambda_{n} \alpha_{n}\|p\|\right] \\
\leq & \left\|x_{n}-p\right\|^{2}+4 \lambda_{n} \alpha_{n}\|p\|\left\|x_{n}-p\right\|+4 \lambda_{n}^{2} \alpha_{n}^{2}\|p\|^{2} \\
= & \left(\left\|x_{n}-p\right\|+2 \lambda_{n} \alpha_{n}\|p\|\right)^{2} . \\
&
\end{aligned}
$$

Hence, it follows from (46), (49), and (52) that

$$
\begin{aligned}
\| y_{n}- & p \| \\
& =\left\|\sigma_{n}\left(x_{n}-p\right)+\tau_{n}\left(\bar{u}_{n}-p\right)+\left(1-\sigma_{n}-\tau_{n}\right)\left(u_{n}-p\right)\right\| \\
& \leq \sigma_{n}\left\|x_{n}-p\right\|+\tau_{n}\left\|\bar{u}_{n}-p\right\|+\left(1-\sigma_{n}-\tau_{n}\right)\left\|u_{n}-p\right\| \\
& \leq \sigma_{n}\left\|x_{n}-p\right\|+\tau_{n}\left\|\bar{u}_{n}-p\right\|+\left(1-\sigma_{n}-\tau_{n}\right)\left\|z_{n}-p\right\|
\end{aligned}
$$




$$
\begin{aligned}
\leq & \sigma_{n}\left\|x_{n}-p\right\|+\tau_{n}\left(\left\|x_{n}-p\right\|+2 \lambda_{n} \alpha_{n}\|p\|\right) \\
& +\left(1-\sigma_{n}-\tau_{n}\right)\left(\left\|x_{n}-p\right\|+\lambda_{n} \alpha_{n}\|p\|\right) \\
= & \left\|x_{n}-p\right\|+\left[2 \tau_{n}+\left(1-\sigma_{n}-\tau_{n}\right)\right] \lambda_{n} \alpha_{n}\|p\| \\
\leq & \left\|x_{n}-p\right\|+2 \lambda_{n} \alpha_{n}\|p\| .
\end{aligned}
$$

Since $\left(\gamma_{n}+\delta_{n}\right) k \leq \gamma_{n}$ for all $n \geq 0$, utilizing Lemma 18, we obtain from (53)

$$
\begin{aligned}
&\left\|x_{n+1}-p\right\| \\
&=\left\|\beta_{n}\left(x_{n}-p\right)+\gamma_{n}\left(y_{n}-p\right)+\delta_{n}\left(S y_{n}-p\right)\right\| \\
& \leq \beta_{n}\left\|x_{n}-p\right\|+\left\|\gamma_{n}\left(y_{n}-p\right)+\delta_{n}\left(S y_{n}-p\right)\right\| \\
& \leq \beta_{n}\left\|x_{n}-p\right\|+\left(\gamma_{n}+\delta_{n}\right)\left\|y_{n}-p\right\| \\
& \leq \beta_{n}\left\|x_{n}-p\right\|+\left(\gamma_{n}+\delta_{n}\right) \\
& \times\left[\left\|x_{n}-p\right\|+2 \lambda_{n} \alpha_{n}\|p\|\right] \\
& \leq\left\|x_{n}-p\right\|+2 b\|p\| \alpha_{n} .
\end{aligned}
$$

Since $\sum_{n=0}^{\infty} \alpha_{n}<\infty$, it is clear that $\sum_{n=0}^{\infty} 2 b\|p\| \alpha_{n}<\infty$. Thus, by Corollary 17, we conclude that

$$
\lim _{n \rightarrow \infty}\left\|x_{n}-p\right\| \text { exists for each } p \in \operatorname{Fix}(S) \cap \Xi \cap \Gamma \text {, }
$$

and the sequence $\left\{x_{n}\right\}$ is bounded. Taking into account that $P_{C}, \nabla f_{\alpha_{n}}, B_{1}$ and $B_{2}$ are Lipschitz continuous, we can easily see that $\left\{z_{n}\right\},\left\{u_{n}\right\},\left\{\bar{u}_{n}\right\},\left\{y_{n}\right\}$, and $\left\{\widetilde{z}_{n}\right\}$ are bounded, where $\widetilde{z}_{n}=P_{C}\left(z_{n}-\mu_{2} B_{2} z_{n}\right)$ for all $n \geq 0$.

Step 2. Consider $\lim _{n \rightarrow \infty}\left\|B_{2} z_{n}-B_{2} p\right\|=0, \lim _{n \rightarrow \infty} \| B_{1} \widetilde{z}_{n}-$ $B_{1} q \|=0$ and $\lim _{n \rightarrow \infty}\left\|x_{n}-z_{n}\right\|=0$, where $q=P_{C}\left(p-\mu_{2} B_{2} p\right)$.

Indeed, utilizing Lemma 18 and the convexity of $\|\cdot\|^{2}$, we obtain from (16) and (47)-(52) that

$$
\begin{aligned}
\left\|x_{n+1}-p\right\|^{2} & \\
= & \left\|\beta_{n}\left(x_{n}-p\right)+\gamma_{n}\left(y_{n}-p\right)+\delta_{n}\left(S y_{n}-p\right)\right\|^{2} \\
\leq & \beta_{n}\left\|x_{n}-p\right\|^{2}+\left(\gamma_{n}+\delta_{n}\right) \\
& \times\left\|\frac{1}{\gamma_{n}+\delta_{n}}\left[\gamma_{n}\left(y_{n}-p\right)+\delta_{n}\left(S y_{n}-p\right)\right]\right\|^{2} \\
\leq & \beta_{n}\left\|x_{n}-p\right\|^{2}+\left(\gamma_{n}+\delta_{n}\right)\left\|y_{n}-p\right\|^{2}
\end{aligned}
$$

$$
\begin{aligned}
& \leq \beta_{n}\left\|x_{n}-p\right\|^{2}+\left(\gamma_{n}+\delta_{n}\right) \\
& \times\left[\sigma_{n}\left\|x_{n}-p\right\|^{2}+\tau_{n}\left\|\bar{u}_{n}-p\right\|^{2}\right. \\
& \left.+\left(1-\sigma_{n}-\tau_{n}\right)\left\|u_{n}-p\right\|^{2}\right] \\
& \leq \beta_{n}\left\|x_{n}-p\right\|^{2}+\left(\gamma_{n}+\delta_{n}\right) \\
& \times\left\{\sigma_{n}\left\|x_{n}-p\right\|^{2}\right. \\
& +\tau_{n}\left[\left\|x_{n}-p\right\|^{2}+2 \lambda_{n} \alpha_{n}\|p\|\left\|z_{n}-p\right\|\right. \\
& \left.+\left(\lambda_{n}^{2}\left(\alpha_{n}+\|A\|^{2}\right)^{2}-1\right)\left\|x_{n}-z_{n}\right\|^{2}\right] \\
& +\left(1-\sigma_{n}-\tau_{n}\right) \\
& \times\left[\left\|z_{n}-p\right\|^{2}-\mu_{2}\left(2 \beta_{2}-\mu_{2}\right)\left\|B_{2} z_{n}-B_{2} p\right\|^{2}\right. \\
& \left.\left.-\mu_{1}\left(2 \beta_{1}-\mu_{1}\right)\left\|B_{1} \widetilde{z}_{n}-B_{1} q\right\|^{2}\right]\right\} \\
& \leq \beta_{n}\left\|x_{n}-p\right\|^{2}+\left(\gamma_{n}+\delta_{n}\right) \\
& \times\left\{\sigma_{n}\left\|x_{n}-p\right\|^{2}\right. \\
& +\tau_{n}\left[\left\|x_{n}-p\right\|^{2}+2 \lambda_{n} \alpha_{n}\|p\|\left\|z_{n}-p\right\|\right. \\
& \left.+\left(\lambda_{n}^{2}\left(\alpha_{n}+\|A\|^{2}\right)^{2}-1\right)\left\|x_{n}-z_{n}\right\|^{2}\right] \\
& +\left(1-\sigma_{n}-\tau_{n}\right) \\
& \times\left[\left\|x_{n}-p\right\|^{2}+2 \lambda_{n} \alpha_{n}\|p\|\left\|z_{n}-p\right\|\right. \\
& -\mu_{2}\left(2 \beta_{2}-\mu_{2}\right)\left\|B_{2} z_{n}-B_{2} p\right\|^{2} \\
& \left.\left.-\mu_{1}\left(2 \beta_{1}-\mu_{1}\right)\left\|B_{1} \widetilde{z}_{n}-B_{1} q\right\|^{2}\right]\right\} \\
& =\left\|x_{n}-p\right\|^{2}+\left(\gamma_{n}+\delta_{n}\right) \\
& \times\left\{\left(1-\sigma_{n}\right) 2 \lambda_{n} \alpha_{n}\|p\|\left\|z_{n}-p\right\|\right. \\
& +\tau_{n}\left(\lambda_{n}^{2}\left(\alpha_{n}+\|A\|^{2}\right)^{2}-1\right)\left\|x_{n}-z_{n}\right\|^{2} \\
& -\left(1-\sigma_{n}-\tau_{n}\right) \\
& \times\left[\mu_{2}\left(2 \beta_{2}-\mu_{2}\right)\left\|B_{2} z_{n}-B_{2} p\right\|^{2}\right. \\
& \left.\left.+\mu_{1}\left(2 \beta_{1}-\mu_{1}\right)\left\|B_{1} \widetilde{z}_{n}-B_{1} q\right\|^{2}\right]\right\} \\
& \leq\left\|x_{n}-p\right\|^{2}+2 \lambda_{n} \alpha_{n}\|p\|\left\|z_{n}-p\right\| \\
& -\left(\gamma_{n}+\delta_{n}\right)\left\{\tau_{n}\left(1-\lambda_{n}^{2}\left(\alpha_{n}+\|A\|^{2}\right)^{2}\right)\left\|x_{n}-z_{n}\right\|^{2}\right. \\
& +\left(1-\sigma_{n}-\tau_{n}\right) \\
& \times\left[\mu_{2}\left(2 \beta_{2}-\mu_{2}\right)\left\|B_{2} z_{n}-B_{2} p\right\|^{2}\right. \\
& \left.\left.+\mu_{1}\left(2 \beta_{1}-\mu_{1}\right)\left\|B_{1} \widetilde{z}_{n}-B_{1} q\right\|^{2}\right]\right\} \text {. }
\end{aligned}
$$


Therefore,

$$
\begin{gathered}
\left(\gamma_{n}+\delta_{n}\right)\left\{\tau_{n}\left(1-\lambda_{n}^{2}\left(\alpha_{n}+\|A\|^{2}\right)^{2}\right)\left\|x_{n}-z_{n}\right\|^{2}\right. \\
+\left(1-\sigma_{n}-\tau_{n}\right) \\
\times\left[\mu_{2}\left(2 \beta_{2}-\mu_{2}\right)\left\|B_{2} z_{n}-B_{2} p\right\|^{2}\right. \\
\left.\left.\quad+\mu_{1}\left(2 \beta_{1}-\mu_{1}\right)\left\|B_{1} \widetilde{z}_{n}-B_{1} q\right\|^{2}\right]\right\} \\
\leq\left\|x_{n}-p\right\|^{2}-\left\|x_{n+1}-p\right\|^{2}+2 \lambda_{n} \alpha_{n}\|p\|\left\|z_{n}-p\right\| .
\end{gathered}
$$

Since $\alpha_{n} \rightarrow 0, \lim _{n \rightarrow \infty}\left\|x_{n}-p\right\|$ exists, $\lim _{\inf _{n \rightarrow \infty}}\left(\gamma_{n}+\delta_{n}\right)>$ $0,\left\{\lambda_{n}\right\} \subset[a, b]$ and $0<\liminf _{n \rightarrow \infty} \tau_{n} \leq \limsup _{n \rightarrow \infty}\left(\sigma_{n}+\right.$ $\left.\tau_{n}\right)<1$, it follows that

$$
\begin{gathered}
\lim _{n \rightarrow \infty}\left\|x_{n}-z_{n}\right\|=0, \\
\lim _{n \rightarrow \infty}\left\|B_{1} \widetilde{z}_{n}-B_{1} q\right\|=0, \\
\lim _{n \rightarrow \infty}\left\|B_{2} z_{n}-B_{2} p\right\|=0 .
\end{gathered}
$$

Step 3. Consider $\lim _{n \rightarrow \infty}\left\|S y_{n}-y_{n}\right\|=0$. Indeed, observe that

$$
\begin{aligned}
\| \bar{u}_{n}- & z_{n} \| \\
& =\left\|P_{C}\left(x_{n}-\lambda_{n} \nabla f_{\alpha_{n}}\left(z_{n}\right)\right)-P_{C}\left(x_{n}-\lambda_{n} \nabla f_{\alpha_{n}}\left(x_{n}\right)\right)\right\| \\
& \leq\left\|\left(x_{n}-\lambda_{n} \nabla f_{\alpha_{n}}\left(z_{n}\right)\right)-\left(x_{n}-\lambda_{n} \nabla f_{\alpha_{n}}\left(x_{n}\right)\right)\right\| \\
& =\lambda_{n}\left\|\nabla f_{\alpha_{n}}\left(z_{n}\right)-\nabla f_{\alpha_{n}}\left(x_{n}\right)\right\| \\
& \leq \lambda_{n}\left(\alpha_{n}+\|A\|^{2}\right)\left\|z_{n}-x_{n}\right\| .
\end{aligned}
$$

This together with $\left\|z_{n}-x_{n}\right\| \rightarrow 0$ implies that $\lim _{n \rightarrow \infty}\left\|\bar{u}_{n}-z_{n}\right\|=0$ and hence $\lim _{n \rightarrow \infty}\left\|\bar{u}_{n}-x_{n}\right\|=0$. By firm nonexpansiveness of $P_{C}$, we have

$$
\begin{aligned}
& \left\|\tilde{z}_{n}-q\right\|^{2} \\
& =\left\|P_{C}\left(z_{n}-\mu_{2} B_{2} z_{n}\right)-P_{C}\left(p-\mu_{2} B_{2} p\right)\right\|^{2} \\
& \leq\left\langle\left(z_{n}-\mu_{2} B_{2} z_{n}\right)-\left(p-\mu_{2} B_{2} p\right), \widetilde{z}_{n}-q\right\rangle \\
& =\frac{1}{2}\left[\left\|z_{n}-p-\mu_{2}\left(B_{2} z_{n}-B_{2} p\right)\right\|^{2}+\left\|\widetilde{z}_{n}-q\right\|^{2}\right. \\
& \left.\quad \quad-\left\|\left(z_{n}-p\right)-\mu_{2}\left(B_{2} z_{n}-B_{2} p\right)-\left(\widetilde{z}_{n}-q\right)\right\|^{2}\right]
\end{aligned}
$$

$$
\begin{aligned}
& \leq \frac{1}{2}\left[\left\|z_{n}-p\right\|^{2}+\left\|\tilde{z}_{n}-q\right\|^{2}\right. \\
& \left.\quad-\left\|\left(z_{n}-\widetilde{z}_{n}\right)-\mu_{2}\left(B_{2} z_{n}-B_{2} p\right)-(p-q)\right\|^{2}\right] \\
& =\frac{1}{2}\left[\left\|z_{n}-p\right\|^{2}+\left\|\tilde{z}_{n}-q\right\|^{2}-\left\|z_{n}-\widetilde{z}_{n}-(p-q)\right\|^{2}\right. \\
& \quad+2 \mu_{2}\left\langle z_{n}-\widetilde{z}_{n}-(p-q), B_{2} z_{n}-B_{2} p\right\rangle \\
& \left.\quad-\mu_{2}^{2}\left\|B_{2} z_{n}-B_{2} p\right\|^{2}\right] \\
& \leq \frac{1}{2}\left[\left\|z_{n}-p\right\|^{2}+\left\|\widetilde{z}_{n}-q\right\|^{2}\right. \\
& \quad-\left\|z_{n}-\widetilde{z}_{n}-(p-q)\right\|^{2} \\
& \left.\quad+2 \mu_{2}\left\|z_{n}-\widetilde{z}_{n}-(p-q)\right\|\left\|B_{2} z_{n}-B_{2} p\right\|\right],
\end{aligned}
$$

that is,

$$
\begin{aligned}
\left\|\widetilde{z}_{n}-q\right\|^{2} & \\
\leq & \left\|z_{n}-p\right\|^{2}-\left\|z_{n}-\widetilde{z}_{n}-(p-q)\right\|^{2} \\
& \quad+2 \mu_{2}\left\|z_{n}-\widetilde{z}_{n}-(p-q)\right\|\left\|B_{2} z_{n}-B_{2} p\right\| .
\end{aligned}
$$

Moreover, using the argument technique similar to the previous one, we derive

$$
\begin{aligned}
& \left\|u_{n}-p\right\|^{2} \\
& =\left\|P_{C}\left(\widetilde{z}_{n}-\mu_{1} B_{1} \widetilde{z}_{n}\right)-P_{C}\left(q-\mu_{1} B_{1} q\right)\right\|^{2} \\
& \leq\left\langle\left(\widetilde{z}_{n}-\mu_{1} B_{1} \widetilde{z}_{n}\right)-\left(q-\mu_{1} B_{1} q\right), u_{n}-p\right\rangle \\
& =\frac{1}{2}\left[\left\|\widetilde{z}_{n}-q-\mu_{1}\left(B_{1} \widetilde{z}_{n}-B_{1} q\right)\right\|^{2}+\left\|u_{n}-p\right\|^{2}\right. \\
& \left.-\left\|\left(\widetilde{z}_{n}-q\right)-\mu_{1}\left(B_{1} \widetilde{z}_{n}-B_{1} q\right)-\left(u_{n}-p\right)\right\|^{2}\right] \\
& \leq \frac{1}{2}\left[\left\|\widetilde{z}_{n}-q\right\|^{2}+\left\|u_{n}-p\right\|^{2}\right. \\
& \left.-\left\|\left(\widetilde{z}_{n}-u_{n}\right)-\mu_{1}\left(B_{1} \widetilde{z}_{n}-B_{1} q\right)+(p-q)\right\|^{2}\right] \\
& =\frac{1}{2}\left[\left\|\widetilde{z}_{n}-q\right\|^{2}+\left\|u_{n}-p\right\|^{2}\right. \\
& -\left\|\widetilde{z}_{n}-u_{n}+(p-q)\right\|^{2} \\
& +2 \mu_{1}\left\langle\widetilde{z}_{n}-u_{n}+(p-q), B_{1} \widetilde{z}_{n}-B_{1} q\right\rangle \\
& \left.-\mu_{1}^{2}\left\|B_{1} \widetilde{z}_{n}-B_{1} q\right\|^{2}\right] \\
& \leq \frac{1}{2}\left[\left\|\widetilde{z}_{n}-q\right\|^{2}+\left\|u_{n}-p\right\|^{2}-\left\|\widetilde{z}_{n}-u_{n}+(p-q)\right\|^{2}\right. \\
& \left.+2 \mu_{1}\left\|\tilde{z}_{n}-u_{n}+(p-q)\right\|\left\|B_{1} \widetilde{z}_{n}-B_{1} q\right\|\right],
\end{aligned}
$$


that is,

$$
\begin{aligned}
\left\|u_{n}-p\right\|^{2} & \\
\leq & \left\|\widetilde{z}_{n}-q\right\|^{2}-\left\|\tilde{z}_{n}-u_{n}+(p-q)\right\|^{2} \\
& +2 \mu_{1}\left\|\widetilde{z}_{n}-u_{n}+(p-q)\right\|\left\|B_{1} \widetilde{z}_{n}-B_{1} q\right\| .
\end{aligned}
$$

Utilizing (47), (52), (61), and (63), we have

$$
\begin{aligned}
& \left\|y_{n}-p\right\|^{2} \\
& =\| \sigma_{n}\left(x_{n}-p\right)+\tau_{n}\left(\bar{u}_{n}-p\right) \\
& +\left(1-\sigma_{n}-\tau_{n}\right)\left(u_{n}-p\right) \|^{2} \\
& \leq \sigma_{n}\left\|x_{n}-p\right\|^{2}+\tau_{n}\left\|\bar{u}_{n}-p\right\|^{2} \\
& +\left(1-\sigma_{n}-\tau_{n}\right)\left\|u_{n}-p\right\|^{2} \\
& \leq \sigma_{n}\left\|x_{n}-p\right\|^{2} \\
& +\tau_{n}\left(\left\|x_{n}-p\right\|^{2}+2 \lambda_{n} \alpha_{n}\|p\|\left\|z_{n}-p\right\|\right) \\
& +\left(1-\sigma_{n}-\tau_{n}\right) \\
& \times\left[\left\|\widetilde{z}_{n}-q\right\|^{2}-\left\|\widetilde{z}_{n}-u_{n}+(p-q)\right\|^{2}\right. \\
& \left.+2 \mu_{1}\left\|\tilde{z}_{n}-u_{n}+(p-q)\right\|\left\|B_{1} \widetilde{z}_{n}-B_{1} q\right\|\right] \\
& \leq \sigma_{n}\left\|x_{n}-p\right\|^{2}+\tau_{n}\left(\left\|x_{n}-p\right\|^{2}+2 \lambda_{n} \alpha_{n}\|p\|\left\|z_{n}-p\right\|\right) \\
& +\left(1-\sigma_{n}-\tau_{n}\right) \\
& \times\left\{\left\|z_{n}-p\right\|^{2}-\left\|z_{n}-\widetilde{z}_{n}-(p-q)\right\|^{2}\right. \\
& +2 \mu_{2}\left\|z_{n}-\widetilde{z}_{n}-(p-q)\right\|\left\|B_{2} z_{n}-B_{2} p\right\| \\
& -\left\|\tilde{z}_{n}-u_{n}+(p-q)\right\|^{2} \\
& \left.+2 \mu_{1}\left\|\widetilde{z}_{n}-u_{n}+(p-q)\right\|\left\|B_{1} \widetilde{z}_{n}-B_{1} q\right\|\right\} \\
& \leq \sigma_{n}\left\|x_{n}-p\right\|^{2}+\tau_{n}\left(\left\|x_{n}-p\right\|^{2}+2 \lambda_{n} \alpha_{n}\|p\|\left\|z_{n}-p\right\|\right) \\
& +\left(1-\sigma_{n}-\tau_{n}\right) \\
& \times\left\{\left\|x_{n}-p\right\|^{2}+2 \lambda_{n} \alpha_{n}\|p\|\left\|z_{n}-p\right\|\right. \\
& -\left\|z_{n}-\widetilde{z}_{n}-(p-q)\right\|^{2}+2 \mu_{2}\left\|z_{n}-\widetilde{z}_{n}-(p-q)\right\| \\
& \times\left\|B_{2} z_{n}-B_{2} p\right\|-\left\|\widetilde{z}_{n}-u_{n}+(p-q)\right\|^{2} \\
& \left.+2 \mu_{1}\left\|\tilde{z}_{n}-u_{n}+(p-q)\right\|\left\|B_{1} \widetilde{z}_{n}-B_{1} q\right\|\right\} \\
& \leq\left\|x_{n}-p\right\|^{2}+2 \lambda_{n} \alpha_{n}\|p\|\left\|z_{n}-p\right\| \\
& +2 \mu_{2}\left\|z_{n}-\widetilde{z}_{n}-(p-q)\right\|\left\|B_{2} z_{n}-B_{2} p\right\|
\end{aligned}
$$

$+2 \mu_{1}\left\|\tilde{z}_{n}-u_{n}+(p-q)\right\|\left\|B_{1} \tilde{z}_{n}-B_{1} q\right\|$$$
-\left(1-\sigma_{n}-\tau_{n}\right)\left(\left\|z_{n}-\widetilde{z}_{n}-(p-q)\right\|^{2}\right.
$$$$
\left.+\left\|\tilde{z}_{n}-u_{n}+(p-q)\right\|^{2}\right) .
$$

Thus, utilizing Lemma 14, from (16) and (64) it follows that

$$
\begin{aligned}
& \left\|x_{n+1}-p\right\|^{2} \\
& =\left\|\beta_{n}\left(x_{n}-p\right)+\gamma_{n}\left(y_{n}-p\right)+\delta_{n}\left(S y_{n}-p\right)\right\|^{2} \\
& =\| \beta_{n}\left(x_{n}-p\right)+\left(1-\beta_{n}\right) \cdot \frac{1}{1-\beta_{n}} \\
& \times\left[\gamma_{n}\left(y_{n}-p\right)+\delta_{n}\left(S y_{n}-p\right)\right] \|^{2} \\
& =\beta_{n}\left\|x_{n}-p\right\|^{2}+\left(1-\beta_{n}\right) \\
& \times\left\|\frac{1}{1-\beta_{n}}\left[\gamma_{n}\left(y_{n}-p\right)+\delta_{n}\left(S y_{n}-p\right)\right]\right\|^{2} \\
& -\beta_{n}\left(1-\beta_{n}\right) \\
& \times\left\|\frac{1}{1-\beta_{n}}\left[\gamma_{n}\left(x_{n}-y_{n}\right)+\delta_{n}\left(x_{n}-S y_{n}\right)\right]\right\|^{2} \\
& \leq \beta_{n}\left\|x_{n}-p\right\|^{2}+\left(1-\beta_{n}\right)\left\|y_{n}-p\right\|^{2}-\beta_{n}\left(1-\beta_{n}\right) \\
& \times\left\|\frac{1}{1-\beta_{n}}\left[\gamma_{n}\left(x_{n}-y_{n}\right)+\delta_{n}\left(x_{n}-S y_{n}\right)\right]\right\|^{2} \\
& \leq \beta_{n}\left\|x_{n}-p\right\|^{2}+\left(1-\beta_{n}\right) \\
& \times\left\{\left\|x_{n}-p\right\|^{2}+2 \lambda_{n} \alpha_{n}\|p\|\left\|z_{n}-p\right\|\right. \\
& +2 \mu_{2}\left\|z_{n}-\widetilde{z}_{n}-(p-q)\right\|\left\|B_{2} z_{n}-B_{2} p\right\| \\
& +2 \mu_{1}\left\|\widetilde{z}_{n}-u_{n}+(p-q)\right\|\left\|B_{1} \widetilde{z}_{n}-B_{1} q\right\| \\
& -\left(1-\sigma_{n}-\tau_{n}\right)\left(\left\|z_{n}-\widetilde{z}_{n}-(p-q)\right\|^{2}\right. \\
& \left.\left.+\left\|\tilde{z}_{n}-u_{n}+(p-q)\right\|^{2}\right)\right\} \\
& -\beta_{n}\left(1-\beta_{n}\right) \\
& \times\left\|\frac{1}{1-\beta_{n}}\left[\gamma_{n}\left(x_{n}-y_{n}\right)+\delta_{n}\left(x_{n}-S y_{n}\right)\right]\right\|^{2} \\
& \leq\left\|x_{n}-p\right\|^{2}+2 \lambda_{n} \alpha_{n}\|p\|\left\|z_{n}-p\right\| \\
& +2 \mu_{2}\left\|z_{n}-\widetilde{z}_{n}-(p-q)\right\|\left\|B_{2} z_{n}-B_{2} p\right\| \\
& +2 \mu_{1}\left\|\widetilde{z}_{n}-u_{n}+(p-q)\right\|\left\|B_{1} \widetilde{z}_{n}-B_{1} q\right\| \\
& -\left(1-\beta_{n}\right)\left(1-\sigma_{n}-\tau_{n}\right)\left(\left\|z_{n}-\widetilde{z}_{n}-(p-q)\right\|^{2}\right. \\
& \left.+\left\|\tilde{z}_{n}-u_{n}+(p-q)\right\|^{2}\right)
\end{aligned}
$$




$$
\begin{aligned}
& -\beta_{n}\left(1-\beta_{n}\right) \\
& \times\left\|\frac{1}{1-\beta_{n}}\left[\gamma_{n}\left(x_{n}-y_{n}\right)+\delta_{n}\left(x_{n}-S y_{n}\right)\right]\right\|^{2},
\end{aligned}
$$

which hence implies that

$$
\begin{aligned}
& \left(1-\beta_{n}\right)\left(1-\sigma_{n}-\tau_{n}\right) \\
& \quad \times\left(\left\|z_{n}-\widetilde{z}_{n}-(p-q)\right\|^{2}+\left\|\tilde{z}_{n}-u_{n}+(p-q)\right\|^{2}\right) \\
& \quad+\beta_{n}\left(1-\beta_{n}\right)\left\|\frac{1}{1-\beta_{n}}\left[\gamma_{n}\left(x_{n}-y_{n}\right)+\delta_{n}\left(x_{n}-S y_{n}\right)\right]\right\|^{2} \\
& \leq \\
& \quad\left\|x_{n}-p\right\|^{2}-\left\|x_{n+1}-p\right\|^{2}+2 \lambda_{n} \alpha_{n}\|p\|\left\|z_{n}-p\right\| \\
& \quad+2 \mu_{2}\left\|z_{n}-\widetilde{z}_{n}-(p-q)\right\|\left\|B_{2} z_{n}-B_{2} p\right\| \\
& \quad+2 \mu_{1}\left\|\widetilde{z}_{n}-u_{n}+(p-q)\right\|\left\|B_{1} \widetilde{z}_{n}-B_{1} q\right\| .
\end{aligned}
$$

Since $0<\liminf _{n \rightarrow \infty} \beta_{n} \leq \limsup _{n \rightarrow \infty} \beta_{n}<1$, $\lim \sup _{n \rightarrow \infty}\left(\sigma_{n}+\tau_{n}\right)<1,\left\{\lambda_{n}\right\} \subset[a, b], \alpha_{n} \rightarrow 0, \| B_{2} z_{n}-$ $B_{2} p\|\rightarrow 0,\| B_{1} \widetilde{z}_{n}-B_{1} q \| \rightarrow 0$ and $\lim _{n \rightarrow \infty}\left\|x_{n}-p\right\|$ exists, it follows from the boundedness of $\left\{u_{n}\right\},\left\{z_{n}\right\}$ and $\left\{\widetilde{z}_{n}\right\}$ that $\lim _{n \rightarrow \infty}\left\|z_{n}-\widetilde{z}_{n}-(p-q)\right\|=0$,

$$
\begin{aligned}
& \lim _{n \rightarrow \infty}\left\|\tilde{z}_{n}-u_{n}+(p-q)\right\|=0, \\
& \lim _{n \rightarrow \infty}\left\|\gamma_{n}\left(x_{n}-y_{n}\right)+\delta_{n}\left(x_{n}-S y_{n}\right)\right\|=0 .
\end{aligned}
$$

Consequently, it immediately follows that

$$
\lim _{n \rightarrow \infty}\left\|z_{n}-u_{n}\right\|=0, \quad \lim _{n \rightarrow \infty}\left\|u_{n}-\bar{u}_{n}\right\|=0 .
$$

Also, note that

$$
\begin{aligned}
& \left\|y_{n}-\bar{u}_{n}\right\| \\
& \quad \leq \sigma_{n}\left\|x_{n}-\bar{u}_{n}\right\|+\left(1-\sigma_{n}-\tau_{n}\right)\left\|u_{n}-\bar{u}_{n}\right\| \longrightarrow 0 .
\end{aligned}
$$

This together with $\left\|x_{n}-\bar{u}_{n}\right\| \rightarrow 0$ implies that

$$
\lim _{n \rightarrow \infty}\left\|x_{n}-y_{n}\right\|=0 \text {. }
$$

Since

$$
\begin{aligned}
& \left\|\delta_{n}\left(S y_{n}-x_{n}\right)\right\| \\
& \quad=\left\|\gamma_{n}\left(x_{n}-y_{n}\right)+\delta_{n}\left(x_{n}-S y_{n}\right)+\gamma_{n}\left(y_{n}-x_{n}\right)\right\| \\
& \quad \leq\left\|\gamma_{n}\left(x_{n}-y_{n}\right)+\delta_{n}\left(x_{n}-S y_{n}\right)\right\|+\gamma_{n}\left\|x_{n}-y_{n}\right\|,
\end{aligned}
$$

we have

$$
\lim _{n \rightarrow \infty}\left\|S y_{n}-x_{n}\right\|=0, \quad \lim _{n \rightarrow \infty}\left\|S y_{n}-y_{n}\right\|=0 .
$$

Step 4. $\left\{x_{n}\right\},\left\{y_{n}\right\}$, and $\left\{z_{n}\right\}$ converge weakly to an element $\bar{x} \in$ $\operatorname{Fix}(S) \cap \Xi \cap \Gamma$.
Indeed, since $\left\{x_{n}\right\}$ is bounded, there exists a subsequence $\left\{x_{n_{i}}\right\}$ of $\left\{x_{n}\right\}$ that converges weakly to some $\bar{x} \in C$. We obtain that $\bar{x} \in \operatorname{Fix}(S) \cap \Xi \cap \Gamma$. Taking into account that $\left\|x_{n}-y_{n}\right\| \rightarrow 0$ and $\left\|x_{n}-z_{n}\right\| \rightarrow 0$ as $n \rightarrow \infty$, we deduce that $y_{n_{i}} \rightarrow \bar{x}$ weakly and $z_{n_{i}} \rightarrow \bar{x}$ weakly. First, it is clear from Lemma 15 and $\left\|S y_{n}-y_{n}\right\| \rightarrow 0$ that $\bar{x} \in \operatorname{Fix}(S)$. Now let us show that $\bar{x} \in \Xi$. Note that

$$
\begin{aligned}
\| z_{n}- & G\left(z_{n}\right) \| \\
& =\left\|z_{n}-P_{C}\left[P_{C}\left(z_{n}-\mu_{2} B_{2} z_{n}\right)-\mu_{1} B_{1} P_{C}\left(z_{n}-\mu_{2} B_{2} z_{n}\right)\right]\right\| \\
& =\left\|z_{n}-u_{n}\right\| \longrightarrow 0,
\end{aligned}
$$

as $n \rightarrow \infty$ where $G: C \rightarrow C$ is defined as that in Lemma 1 . According to Lemma 15 , we get $\bar{x} \in \Xi$. Further, let us show that $\bar{x} \in \Gamma$. As a matter of fact, define

$$
T v= \begin{cases}\nabla f(v)+N_{C} v, & \text { if } v \in C, \\ \emptyset, & \text { if } v \notin C,\end{cases}
$$

where $N_{C} v=\left\{w \in \mathscr{H}_{1}:\langle v-u, w\rangle \geq 0, \forall u \in C\right\}$. Then, $T$ is maximal monotone and $0 \in T v$ if and only if $v \in \operatorname{VI}(C, \nabla f)$; see [40] for more details. Let $(v, w) \in \operatorname{Gph}(T)$. Then, we have

$$
w \in T v=\nabla f(v)+N_{C} v,
$$

and hence

$$
w-\nabla f(v) \in N_{C} v
$$

So, we have

$$
\langle v-u, w-\nabla f(v)\rangle \geq 0, \quad \forall u \in C .
$$

On the other hand, from

$$
z_{n}=P_{C}\left(x_{n}-\lambda_{n} \nabla f_{\alpha_{n}}\left(x_{n}\right)\right), \quad v \in C,
$$

we have

$$
\left\langle x_{n}-\lambda_{n} \nabla f_{\alpha_{n}}\left(x_{n}\right)-z_{n}, z_{n}-v\right\rangle \geq 0,
$$

and hence,

$$
\left\langle v-z_{n}, \frac{z_{n}-x_{n}}{\lambda_{n}}+\nabla f_{\alpha_{n}}\left(x_{n}\right)\right\rangle \geq 0 .
$$

Therefore, from

$$
w-\nabla f(v) \in N_{C} v, \quad z_{n_{i}} \in C,
$$


we have

$$
\begin{aligned}
&\left\langle v-z_{n_{i}}, w\right\rangle \\
& \geq\left\langle v-z_{n_{i}}, \nabla f(v)\right\rangle \\
& \geq\left\langle v-z_{n_{i}}, \nabla f(v)\right\rangle \\
&-\left\langle v-z_{n_{i}}, \frac{z_{n_{i}}-x_{n_{i}}}{\lambda_{n_{i}}}+\nabla f_{\alpha_{n_{i}}}\left(x_{n_{i}}\right)\right\rangle \\
&=\left\langle v-z_{n_{i}}, \nabla f(v)\right\rangle \\
&-\left\langle v-z_{n_{i}}, \frac{z_{n_{i}}-x_{n_{i}}}{\lambda_{n_{i}}}+\nabla f\left(x_{n_{i}}\right)\right\rangle \\
&-\alpha_{n_{i}}\left\langle v-z_{n_{i}}, x_{n_{i}}\right\rangle \\
&=\left\langle v-z_{n_{i}}, \nabla f(v)-\nabla f\left(z_{n_{i}}\right)\right\rangle \\
&+\left\langle v-z_{n_{i}}, \nabla f\left(z_{n_{i}}\right)-\nabla f\left(x_{n_{i}}\right)\right\rangle \\
&-\left\langle v-z_{n_{i}}, \frac{z_{n_{i}}-x_{n_{i}}}{\lambda_{n_{i}}}\right\rangle-\alpha_{n_{i}}\left\langle v-z_{n_{i}}, x_{n_{i}}\right\rangle \\
& \geq\left\langle v-z_{n_{i}}, \nabla f\left(z_{n_{i}}\right)-\nabla f\left(x_{n_{i}}\right)\right\rangle \\
&-\left\langle v-z_{n_{i}}, \frac{z_{n_{i}}-x_{n_{i}}}{\lambda_{n_{i}}}\right\rangle-\alpha_{n_{i}}\left\langle v-z_{n_{i}}, x_{n_{i}}\right\rangle .
\end{aligned}
$$

Hence, we get

$$
\langle v-\bar{x}, w\rangle \geq 0, \quad \text { as } i \longrightarrow \infty .
$$

Since $T$ is maximal monotone, we have $\bar{x} \in T^{-1} 0$, and hence, $\bar{x} \in \operatorname{VI}(C, \nabla f)$. Thus, it is clear that $\bar{x} \in \Gamma$. Therefore, $\bar{x} \in$ $\operatorname{Fix}(S) \cap \Xi \cap \Gamma$.

Let $\left\{x_{n_{j}}\right\}$ be another subsequence of $\left\{x_{n}\right\}$ such that $\left\{x_{n_{j}}\right\}$ converges weakly to $\hat{x} \in C$. Then, $\hat{x} \in \operatorname{Fix}(S) \cap \Xi \cap \Gamma$. Let us show that $\bar{x}=\widehat{x}$. Assume that $\bar{x} \neq \hat{x}$. From the Opial condition [41], we have

$$
\begin{aligned}
\lim _{n \rightarrow \infty} & \left\|x_{n}-\bar{x}\right\| \\
= & \liminf _{i \rightarrow \infty}\left\|x_{n_{i}}-\bar{x}\right\|<\liminf _{i \rightarrow \infty}\left\|x_{n_{i}}-\hat{x}\right\| \\
= & \liminf _{n \rightarrow \infty}\left\|x_{n}-\hat{x}\right\|=\liminf _{j \rightarrow \infty}\left\|x_{n_{j}}-\hat{x}\right\| \\
< & \liminf _{j \rightarrow \infty}\left\|x_{n_{j}}-\bar{x}\right\|=\lim _{n \rightarrow \infty}\left\|x_{n}-\bar{x}\right\| .
\end{aligned}
$$

This leads to a contradiction. Consequently, we have $\bar{x}=\widehat{x}$. This implies that $\left\{x_{n}\right\}$ converges weakly to $\bar{x} \in \operatorname{Fix}(S) \cap \Xi \cap \Gamma$. Further, from $\left\|x_{n}-y_{n}\right\| \rightarrow 0$ and $\left\|x_{n}-z_{n}\right\| \rightarrow 0$, it follows that both $\left\{y_{n}\right\}$ and $\left\{z_{n}\right\}$ converge weakly to $\bar{x}$. This completes the proof.

Corollary 21. Let $C$ be a nonempty closed convex subset of a real Hilbert space $\mathscr{H}_{1}$. Let $A \in B\left(\mathscr{H}_{1}, \mathscr{H}_{2}\right)$ and $B_{i}: C \rightarrow$
$\mathscr{H}_{1}$ be $\beta_{i}$-inverse strongly monotone for $i=1$, 2. Let $S$ : $C \rightarrow C$ be a $k$-strictly pseudocontractive mapping such that $\operatorname{Fix}(S) \cap \Xi \cap \Gamma \neq \emptyset$. For given $x_{0} \in C$ arbitrarily, let the sequences $\left\{x_{n}\right\},\left\{y_{n}\right\},\left\{z_{n}\right\}$ be generated iteratively by

$$
\begin{aligned}
z_{n}= & P_{C}\left(x_{n}-\lambda_{n} \nabla f_{\alpha_{n}}\left(x_{n}\right)\right), \\
y_{n}= & \tau_{n} P_{C}\left(x_{n}-\lambda_{n} \nabla f_{\alpha_{n}}\left(z_{n}\right)\right) \\
& +\left(1-\tau_{n}\right) P_{C}\left[P_{C}\left(z_{n}-\mu_{2} B_{2} z_{n}\right)\right. \\
& \left.-\mu_{1} B_{1} P_{C}\left(z_{n}-\mu_{2} B_{2} z_{n}\right)\right], \\
x_{n+1}= & \beta_{n} x_{n}+\gamma_{n} y_{n}+\delta_{n} S y_{n}, \quad \forall n \geq 0,
\end{aligned}
$$

where $\mu_{i} \in\left(0,2 \beta_{i}\right)$ for $i=1,2,\left\{\alpha_{n}\right\} \subset(0, \infty),\left\{\lambda_{n}\right\} \subset(0,1 /$ $\left.\|A\|^{2}\right)$ and $\left\{\tau_{n}\right\},\left\{\beta_{n}\right\},\left\{\gamma_{n}\right\},\left\{\delta_{n}\right\} \subset[0,1]$ such that

(i) $\sum_{n=0}^{\infty} \alpha_{n}<\infty$;

(ii) $\beta_{n}+\gamma_{n}+\delta_{n}=1$ and $\left(\gamma_{n}+\delta_{n}\right) k \leq \gamma_{n}$ for all $n \geq 0$;

(iii) $0<\liminf _{n \rightarrow \infty} \tau_{n} \leq \limsup \sup _{n \rightarrow \infty} \tau_{n}<1$;

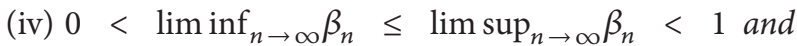
$\liminf _{n \rightarrow \infty} \delta_{n}>0$;

(v) $0<\liminf \operatorname{in}_{n \rightarrow \infty} \lambda_{n} \leq \lim \sup _{n \rightarrow \infty} \lambda_{n}<1 /\|A\|^{2}$.

Then all the sequences $\left\{x_{n}\right\},\left\{y_{n}\right\},\left\{z_{n}\right\}$ converge weakly to an element $\bar{x} \in \operatorname{Fix}(S) \cap \Xi \cap \Gamma$. Furthermore, $(\bar{x}, \bar{y})$ is a solution of the GSVI (3), where $\bar{y}=P_{C}\left(\bar{x}-\mu_{2} B_{2} \bar{x}\right)$.

Proof. In Theorem 20, put $\sigma_{n}=0$ for all $n \geq 0$. Then, in this case, Theorem 20 reduces to Corollary 21.

Next, utilizing Corollary 21, we give the following result.

Corollary 22. Let $C$ be a nonempty closed convex subset of a real Hilbert space $\mathscr{H}_{1}$. Let $A \in B\left(\mathscr{H}_{1}, \mathscr{H}_{2}\right)$ and $S: C \rightarrow$ $C$ be a nonexpansive mapping such that $\operatorname{Fix}(S) \cap \Gamma \neq \emptyset$. For given $x_{0} \in C$ arbitrarily, let the sequences $\left\{x_{n}\right\},\left\{y_{n}\right\},\left\{z_{n}\right\}$ be generated iteratively by

$$
\begin{gathered}
z_{n}=P_{C}\left(x_{n}-\lambda_{n} \nabla f_{\alpha_{n}}\left(x_{n}\right)\right), \\
y_{n}=\left(1-\tau_{n}\right) z_{n}+\tau_{n} P_{C}\left(x_{n}-\lambda_{n} \nabla f_{\alpha_{n}}\left(z_{n}\right)\right), \\
x_{n+1}=\beta_{n} x_{n}+\left(1-\beta_{n}\right) S y_{n}, \quad \forall n \geq 0,
\end{gathered}
$$

where $\left\{\alpha_{n}\right\} \subset(0, \infty),\left\{\lambda_{n}\right\} \subset\left(0,1 /\|A\|^{2}\right)$ and $\left\{\tau_{n}\right\},\left\{\beta_{n}\right\} \subset[0,1]$ such that
(i) $\sum_{n=0}^{\infty} \alpha_{n}<\infty$;
(ii) $0<\liminf _{n \rightarrow \infty} \tau_{n} \leq \lim \sup _{n \rightarrow \infty} \tau_{n}<1$;
(iii) $0<\liminf _{n \rightarrow \infty} \beta_{n} \leq \lim \sup _{n \rightarrow \infty} \beta_{n}<1$;
(iv) $0<\liminf _{n \rightarrow \infty} \lambda_{n} \leq \lim \sup _{n \rightarrow \infty} \lambda_{n}<1 /\|A\|^{2}$.

Then all the sequences $\left\{x_{n}\right\},\left\{y_{n}\right\},\left\{z_{n}\right\}$ converge weakly to an element $\bar{x} \in \operatorname{Fix}(S) \cap \Gamma$. 
Proof. In Corollary 21, put $B_{1}=B_{2}=0$ and $\gamma_{n}=0$. Then, $\Xi=C, \beta_{n}+\delta_{n}=1$ for all $n \geq 0$, and the iterative scheme (85) is equivalent to

$$
\begin{gathered}
z_{n}=P_{C}\left(x_{n}-\lambda_{n} \nabla f_{\alpha_{n}}\left(x_{n}\right)\right), \\
y_{n}=\tau_{n} P_{C}\left(x_{n}-\lambda_{n} \nabla f_{\alpha_{n}}\left(z_{n}\right)\right)+\left(1-\tau_{n}\right) z_{n}, \\
x_{n+1}=\beta_{n} x_{n}+\delta_{n} S y_{n}, \quad \forall n \geq 0 .
\end{gathered}
$$

This is equivalent to (86). Since $S$ is a nonexpansive mapping, $S$ must be a $k$-strictly pseudocontractive mapping with $k=0$. In this case, it is easy to see that all the conditions (i)-(v) in Corollary 21 are satisfied. Therefore, in terms of Corollary 21, we obtain the desired result.

Now, we are in a position to prove the weak convergence of the sequences generated by the Mann-type extragradient iterative algorithm (17) with regularization.

Theorem 23. Let $C$ be a nonempty closed convex subset of a real Hilbert space $\mathscr{H}_{1}$. Let $A \in B\left(\mathscr{H}_{1}, \mathscr{H}_{2}\right)$ and $B_{i}$ : $C \rightarrow \mathscr{H}_{1}$ be $\beta_{i}$-inverse strongly monotone for $i=1,2$. Let $S: C \rightarrow C$ be a $k$-strictly pseudocontractive mapping such that $\operatorname{Fix}(S) \cap \Xi \cap \Gamma \neq \emptyset$. For given $x_{0} \in C$ arbitrarily, let $\left\{x_{n}\right\},\left\{u_{n}\right\},\left\{\widetilde{u}_{n}\right\}$ be the sequences generated by the Manntype extragradient iterative algorithm (17) with regularization, where $\mu_{i} \in\left(0,2 \beta_{i}\right)$ for $i=1,2,\left\{\alpha_{n}\right\} \subset(0, \infty),\left\{\lambda_{n}\right\} \subset$ $\left(0,1 /\|A\|^{2}\right)$ and $\left\{\sigma_{n}\right\},\left\{\beta_{n}\right\},\left\{\gamma_{n}\right\},\left\{\delta_{n}\right\} \subset[0,1]$ such that

(i) $\sum_{n=0}^{\infty} \alpha_{n}<\infty$;

(ii) $\beta_{n}+\gamma_{n}+\delta_{n}=1$ and $\left(\gamma_{n}+\delta_{n}\right) k \leq \gamma_{n}$ for all $n \geq 0$;

(iii) $\limsup _{n \rightarrow \infty} \sigma_{n}<1$;

(iv) $0<\liminf _{n \rightarrow \infty} \beta_{n} \leq \limsup _{n \rightarrow \infty} \beta_{n}<1$ and $\liminf _{n \rightarrow \infty} \delta_{n}>0$;

(v) $0<\liminf _{n \rightarrow \infty} \lambda_{n} \leq \lim \sup _{n \rightarrow \infty} \lambda_{n}<1 /\|A\|^{2}$.

Then the sequences $\left\{x_{n}\right\},\left\{u_{n}\right\},\left\{\tilde{u}_{n}\right\}$ converge weakly to an element $\bar{x} \in \operatorname{Fix}(S) \cap \Xi \cap \Gamma$. Furthermore, $(\bar{x}, \bar{y})$ is a solution of the GSVI (3), where $\bar{y}=P_{C}\left(\bar{x}-\mu_{2} B_{2} \bar{x}\right)$.

Proof. First, taking into account $0<\liminf _{n \rightarrow \infty} \lambda_{n} \leq$ $\lim \sup _{n \rightarrow \infty} \lambda_{n}<1 /\|A\|^{2}$, without loss of generality, we may assume that $\left\{\lambda_{n}\right\} \subset[a, b]$ for some $a, b \in\left(0,1 /\|A\|^{2}\right)$. Repeating the same argument as that in the proof of Theorem 20, we can show that $P_{C}\left(I-\lambda \nabla f_{\alpha}\right)$ is $\zeta$-averaged for each $\lambda \in\left(0,1 /\left(\alpha+\|A\|^{2}\right)\right)$, where $\zeta=\left(2+\lambda\left(\alpha+\|A\|^{2}\right)\right) / 4$. Further, repeating the same argument as that in the proof of Theorem 20, we can also show that for each integer $n \geq 0$, $P_{C}\left(I-\lambda_{n} \nabla f_{\alpha_{n}}\right)$ is $\zeta_{n}$-averaged with $\zeta_{n}=\left(2+\lambda_{n}\left(\alpha_{n}+\|A\|^{2}\right)\right) / 4 \epsilon$ $(0,1)$.

Next we divide the remainder of the proof into several steps.

Step 1. $\left\{x_{n}\right\}$ is bounded.

Indeed, take $p \in \operatorname{Fix}(S) \cap \Xi \cap \Gamma$ arbitrarily. Then $S p=p$, $P_{C}(I-\lambda \nabla f) p=p$ for $\lambda \in\left(0,2 /\|A\|^{2}\right)$, and

$$
p=P_{C}\left[P_{C}\left(p-\mu_{2} B_{2} p\right)-\mu_{1} B_{1} P_{C}\left(p-\mu_{2} B_{2} p\right)\right] .
$$

For simplicity, we write

$$
\begin{gathered}
q=P_{C}\left(p-\mu_{2} B_{2} p\right), \\
\tilde{x}_{n}=P_{C}\left(x_{n}-\mu_{2} B_{2} x_{n}\right), \\
\bar{u}_{n}=P_{C}\left(u_{n}-\lambda_{n} \nabla f_{\alpha_{n}}\left(\tilde{u}_{n}\right)\right),
\end{gathered}
$$

for each $n \geq 0$. Then $y_{n}=\sigma_{n} x_{n}+\left(1-\sigma_{n}\right) \bar{u}_{n}$ for each $n \geq 0$. Utilizing the arguments similar to those of (46) and (47) in the proof of Theorem 20, from (17) we can obtain

$$
\begin{aligned}
\left\|\tilde{u}_{n}-p\right\| & \leq\left\|u_{n}-p\right\|+\lambda_{n} \alpha_{n}\|p\|, \\
\left\|\tilde{u}_{n}-p\right\|^{2} & \leq\left\|u_{n}-p\right\|^{2}+2 \lambda_{n} \alpha_{n}\|p\|\left\|\tilde{u}_{n}-p\right\| .
\end{aligned}
$$

Since $B_{i}: C \rightarrow \mathscr{H}_{1}$ is $\beta_{i}$-inverse strongly monotone and $0<\mu_{i}<2 \beta_{i}$ for $i=1,2$, utilizing the argument similar to that of (49) in the proof of Theorem 20, we can obtain that for all $n \geq 0$,

$$
\begin{aligned}
\| u_{n}- & p \|^{2} \\
\leq & \left\|x_{n}-p\right\|^{2}-\mu_{2}\left(2 \beta_{2}-\mu_{2}\right)\left\|B_{2} x_{n}-B_{2} p\right\|^{2} \\
& \quad-\mu_{1}\left(2 \beta_{1}-\mu_{1}\right)\left\|B_{1} \tilde{x}_{n}-B_{1} q\right\|^{2} \leq\left\|x_{n}-p\right\|^{2} .
\end{aligned}
$$

Utilizing the argument similar to that of (52) in the proof of Theorem 20, from (90) we can obtain

$$
\begin{aligned}
\| \bar{u}_{n}- & p \|^{2} \\
\leq & \left\|u_{n}-p\right\|^{2}+2 \lambda_{n} \alpha_{n}\|p\|\left\|\tilde{u}_{n}-p\right\| \\
& +\left(\lambda_{n}^{2}\left(\alpha_{n}+\|A\|^{2}\right)^{2}-1\right)\left\|u_{n}-\widetilde{u}_{n}\right\|^{2} \\
\leq & \left(\left\|u_{n}-p\right\|+2 \lambda_{n} \alpha_{n}\|p\|\right)^{2} .
\end{aligned}
$$

Hence, it follows from (92) and (93) that

$$
\begin{aligned}
\| y_{n}- & p \| \\
= & \left\|\sigma_{n}\left(x_{n}-p\right)+\left(1-\sigma_{n}\right)\left(\bar{u}_{n}-p\right)\right\| \\
\leq & \sigma_{n}\left\|x_{n}-p\right\|+\left(1-\sigma_{n}\right)\left\|\bar{u}_{n}-p\right\| \\
\leq & \sigma_{n}\left\|x_{n}-p\right\|+\left(1-\sigma_{n}\right) \\
& \times\left(\left\|x_{n}-p\right\|+2 \lambda_{n} \alpha_{n}\|p\|\right) \\
\leq & \left\|x_{n}-p\right\|+2 \lambda_{n} \alpha_{n}\|p\| .
\end{aligned}
$$

Since $\left(\gamma_{n}+\delta_{n}\right) k \leq \gamma_{n}$ for all $n \geq 0$, by Lemma 18 we can readily see from (94) that

$$
\begin{aligned}
\| x_{n+1} & -p \| \\
& \leq \beta_{n}\left\|x_{n}-p\right\|+\left(\gamma_{n}+\delta_{n}\right)\left\|y_{n}-p\right\| \\
& \leq\left\|x_{n}-p\right\|+2 b\|p\| \alpha_{n} .
\end{aligned}
$$

Since $\sum_{n=0}^{\infty} \alpha_{n}<\infty$, it is clear that $\sum_{n=0}^{\infty} 2 b\|p\| \alpha_{n}<\infty$. Thus, by Corollary 17 we conclude that

$\lim _{n \rightarrow \infty}\left\|x_{n}-p\right\|$ exists for each $p \in \operatorname{Fix}(S) \cap \Xi \cap \Gamma$, 
and the sequence $\left\{x_{n}\right\}$ is bounded. Since $P_{C}, \nabla f_{\alpha_{n}}, B_{1}$ and $B_{2}$ are Lipschitz continuous, it is easy to see that $\left\{u_{n}\right\},\left\{\widetilde{u}_{n}\right\},\left\{\bar{u}_{n}\right\}$, $\left\{y_{n}\right\}$ and $\left\{\tilde{x}_{n}\right\}$ are bounded, where $\tilde{x}_{n}=P_{C}\left(x_{n}-\mu_{2} B_{2} x_{n}\right)$ for all $n \geq 0$.

Step 2. Consider $\lim _{n \rightarrow \infty}\left\|B_{2} x_{n}-B_{2} p\right\|=0, \lim _{n \rightarrow \infty} \| B_{1} \widetilde{x}_{n}-$ $B_{1} q \|=0$ and $\lim _{n \rightarrow \infty}\left\|u_{n}-\widetilde{u}_{n}\right\|=0$, where $q=P_{C}\left(p-\mu_{2} B_{2} p\right)$.

Indeed, utilizing Lemma 18 and the convexity of $\|\cdot\|^{2}$, we obtain from (17), (92), and (93) that

$$
\begin{aligned}
& \left\|x_{n+1}-p\right\|^{2} \\
& \leq \beta_{n}\left\|x_{n}-p\right\|^{2}+\left(\gamma_{n}+\delta_{n}\right)\left\|y_{n}-p\right\|^{2} \\
& \leq \beta_{n}\left\|x_{n}-p\right\|^{2}+\left(\gamma_{n}+\delta_{n}\right) \\
& \quad \times\left[\sigma_{n}\left\|x_{n}-p\right\|^{2}+\left(1-\sigma_{n}\right)\left\|\bar{u}_{n}-p\right\|^{2}\right] \\
& \leq \beta_{n}\left\|x_{n}-p\right\|^{2}+\left(\gamma_{n}+\delta_{n}\right) \\
& \times\left\{\sigma_{n}\left\|x_{n}-p\right\|^{2}+\left(1-\sigma_{n}\right)\right. \\
& \quad \times\left[\left\|u_{n}-p\right\|^{2}+2 \lambda_{n} \alpha_{n}\|p\|\left\|\widetilde{u}_{n}-p\right\|\right. \\
& \left.\left.\quad+\left(\lambda_{n}^{2}\left(\alpha_{n}+\|A\|^{2}\right)^{2}-1\right)\left\|u_{n}-\widetilde{u}_{n}\right\|^{2}\right]\right\} \\
& \leq \beta_{n}\left\|x_{n}-p\right\|^{2}+\left(\gamma_{n}+\delta_{n}\right) \\
& \times\left\{\sigma_{n}\left\|x_{n}-p\right\|^{2}+\left(1-\sigma_{n}\right)\right. \\
& \quad \times\left[\left\|x_{n}-p\right\|^{2}-\mu_{2}\left(2 \beta_{2}-\mu_{2}\right)\left\|B_{2} x_{n}-B_{2} p\right\|^{2}\right. \\
& \quad \quad-\mu_{1}\left(2 \beta_{1}-\mu_{1}\right)\left\|B_{1} \tilde{x}_{n}-B_{1} q\right\|^{2}+2 \lambda_{n} \alpha_{n}\|p\|\left\|\widetilde{u}_{n}-p\right\| \\
& \left.\left.\quad+\left(\lambda_{n}^{2}\left(\alpha_{n}+\|A\|^{2}\right)^{2}-1\right)\left\|u_{n}-\widetilde{u}_{n}\right\|^{2}\right]\right\} \\
& \left.\quad+\left(1-\lambda_{n}^{2}\left(\alpha_{n}+\|A\|^{2}\right)^{2}\right)\left\|u_{n}-\widetilde{u}_{n}\right\|^{2}\right\} . \\
& \times\left\{x_{n}-p\left\|^{2}+2 \lambda_{n} \alpha_{n}\right\| p\|\| \widetilde{u}_{n}-p \|-\left(\gamma_{n}+\delta_{n}\right)\left(1-\sigma_{n}\right)\right. \\
& \times\left\{\mu_{2}\left(2 \beta_{2}-\mu_{2}\right)\left\|B_{2} x_{n}-B_{2} p\right\|^{2}\right. \\
& +\mu_{1}\left(2 \beta_{1}-\mu_{1}\right)\left\|B_{1} \tilde{x}_{n}-B_{1} q\right\|^{2} \\
& \quad
\end{aligned}
$$

Therefore,

$$
\begin{aligned}
\left(\gamma_{n}+\delta_{n}\right)\left(1-\sigma_{n}\right) & \left\{\mu_{2}\left(2 \beta_{2}-\mu_{2}\right)\left\|B_{2} x_{n}-B_{2} p\right\|^{2}\right. \\
& +\mu_{1}\left(2 \beta_{1}-\mu_{1}\right)\left\|B_{1} \widetilde{x}_{n}-B_{1} q\right\|^{2} \\
& \left.+\left(1-\lambda_{n}^{2}\left(\alpha_{n}+\|A\|^{2}\right)^{2}\right)\left\|u_{n}-\widetilde{u}_{n}\right\|^{2}\right\} \\
\leq\left\|x_{n}-p\right\|^{2}-\| & x_{n+1}-p\left\|^{2}+2 \lambda_{n} \alpha_{n}\right\| p\|\| \widetilde{u}_{n}-p \| .
\end{aligned}
$$

Since $\alpha_{n} \rightarrow 0, \lim \sup _{n \rightarrow \infty} \sigma_{n}<1, \lim _{n \rightarrow \infty}\left\|x_{n}-p\right\|$ exists, $\liminf _{n \rightarrow \infty}\left(\gamma_{n}+\delta_{n}\right)>0$ and $\left\{\lambda_{n}\right\} \subset[a, b]$ for some $a, b \in$ $\left(0,1 /\|A\|^{2}\right)$, it follows from the boundedness of $\left\{\widetilde{u}_{n}\right\}$ that

$$
\begin{aligned}
& \lim _{n \rightarrow \infty}\left\|u_{n}-\tilde{u}_{n}\right\|=0, \\
& \lim _{n \rightarrow \infty}\left\|B_{1} \tilde{x}_{n}-B_{1} q\right\|=0, \\
& \lim _{n \rightarrow \infty}\left\|B_{2} x_{n}-B_{2} p\right\|=0 .
\end{aligned}
$$

Step 3. Consider $\lim _{n \rightarrow \infty}\left\|S y_{n}-y_{n}\right\|=0$.

Indeed, utilizing the Lipschitz continuity of $\nabla f_{\alpha_{n}}$, we have

$$
\begin{aligned}
\| \bar{u}_{n}- & \tilde{u}_{n} \| \\
& =\left\|P_{C}\left(u_{n}-\lambda_{n} \nabla f_{\alpha_{n}}\left(\widetilde{u}_{n}\right)\right)-P_{C}\left(u_{n}-\lambda_{n} \nabla f_{\alpha_{n}}\left(u_{n}\right)\right)\right\| \\
& \leq \lambda_{n}\left(\alpha_{n}+\|A\|^{2}\right)\left\|\tilde{u}_{n}-u_{n}\right\| .
\end{aligned}
$$

This together with $\left\|\widetilde{u}_{n}-u_{n}\right\| \rightarrow 0$ implies that $\lim _{n \rightarrow \infty} \| \bar{u}_{n}-$ $\widetilde{u}_{n} \|=0$ and hence $\lim _{n \rightarrow \infty}\left\|\bar{u}_{n}-u_{n}\right\|=0$. Utilizing the arguments similar to those of (61) and (63) in the proof of Theorem 20, we get

$$
\begin{aligned}
\left\|\tilde{x}_{n}-q\right\|^{2} \leq & \left\|x_{n}-p\right\|^{2}-\left\|x_{n}-\widetilde{x}_{n}-(p-q)\right\|^{2} \\
& +2 \mu_{2}\left\|x_{n}-\widetilde{x}_{n}-(p-q)\right\|\left\|B_{2} x_{n}-B_{2} p\right\|, \\
\left\|u_{n}-p\right\|^{2} \leq & \left\|\widetilde{x}_{n}-q\right\|^{2}-\left\|\widetilde{x}_{n}-u_{n}+(p-q)\right\|^{2} \\
& +2 \mu_{1}\left\|\widetilde{x}_{n}-u_{n}+(p-q)\right\|\left\|B_{1} \widetilde{x}_{n}-B_{1} q\right\| .
\end{aligned}
$$

Utilizing (91) and (101), we have

$$
\begin{aligned}
\| \bar{u}_{n}- & p \|^{2} \\
= & \left\|\widetilde{u}_{n}-p+\bar{u}_{n}-\widetilde{u}_{n}\right\|^{2} \\
\leq & \left\|\widetilde{u}_{n}-p\right\|^{2}+2\left\langle\bar{u}_{n}-\widetilde{u}_{n}, \bar{u}_{n}-p\right\rangle \\
\leq & \left\|\widetilde{u}_{n}-p\right\|^{2}+2\left\|\bar{u}_{n}-\widetilde{u}_{n}\right\|\left\|\bar{u}_{n}-p\right\| \\
\leq & \left\|u_{n}-p\right\|^{2}+2 \lambda_{n} \alpha_{n}\|p\|\left\|\tilde{u}_{n}-p\right\| \\
& +2\left\|\bar{u}_{n}-\widetilde{u}_{n}\right\|\left\|\bar{u}_{n}-p\right\| \\
\leq & \left\|\widetilde{x}_{n}-q\right\|^{2}-\left\|\widetilde{x}_{n}-u_{n}+(p-q)\right\|^{2} \\
& +2 \mu_{1}\left\|\widetilde{x}_{n}-u_{n}+(p-q)\right\|\left\|B_{1} \widetilde{x}_{n}-B_{1} q\right\| \\
& +2 \lambda_{n} \alpha_{n}\|p\|\left\|\widetilde{u}_{n}-p\right\|+2\left\|\bar{u}_{n}-\widetilde{u}_{n}\right\|\left\|\bar{u}_{n}-p\right\| \\
\leq & \left\|x_{n}-p\right\|^{2}-\left\|x_{n}-\widetilde{x}_{n}-(p-q)\right\|^{2} \\
& +2 \mu_{2}\left\|x_{n}-\widetilde{x}_{n}-(p-q)\right\|\left\|B_{2} x_{n}-B_{2} p\right\| \\
& -\left\|\widetilde{x}_{n}-u_{n}+(p-q)\right\|^{2} \\
& +2 \mu_{1}\left\|\widetilde{x}_{n}-u_{n}+(p-q)\right\|\left\|B_{1} \widetilde{x}_{n}-B_{1} q\right\| \\
& +2 \lambda_{n} \alpha_{n}\|p\|\left\|\widetilde{u}_{n}-p\right\|+2\left\|\bar{u}_{n}-\widetilde{u}_{n}\right\|\left\|\bar{u}_{n}-p\right\| .
\end{aligned}
$$


Thus, utilizing Lemma 14, from (17) and (102), it follows that

$$
\begin{aligned}
& \left\|x_{n+1}-p\right\|^{2} \\
& \leq \beta_{n}\left\|x_{n}-p\right\|^{2}+\left(1-\beta_{n}\right)\left\|y_{n}-p\right\|^{2}-\beta_{n}\left(1-\beta_{n}\right) \\
& \quad \times\left\|\frac{1}{1-\beta_{n}}\left[\gamma_{n}\left(x_{n}-y_{n}\right)+\delta_{n}\left(x_{n}-S y_{n}\right)\right]\right\|^{2} \\
& \leq \beta_{n}\left\|x_{n}-p\right\|^{2}+\left(1-\beta_{n}\right) \\
& \times\left[\sigma_{n}\left\|x_{n}-p\right\|^{2}+\left(1-\sigma_{n}\right)\left\|\bar{u}_{n}-p\right\|^{2}\right] \\
& -\beta_{n}\left(1-\beta_{n}\right)\left\|\frac{1}{1-\beta_{n}}\left[\gamma_{n}\left(x_{n}-y_{n}\right)+\delta_{n}\left(x_{n}-S y_{n}\right)\right]\right\|^{2} \\
& \leq \beta_{n}\left\|x_{n}-p\right\|^{2}+\left(1-\beta_{n}\right) \\
& \times\left\{\sigma_{n}\left\|x_{n}-p\right\|^{2}\right. \\
& \quad+\left(1-\sigma_{n}\right)\left[\left\|x_{n}-p\right\|^{2}-\left\|x_{n}-\tilde{x}_{n}-(p-q)\right\|^{2}\right. \\
& \quad+2 \mu_{2}\left\|x_{n}-\tilde{x}_{n}-(p-q)\right\|\left\|B_{2} x_{n}-B_{2} p\right\| \\
& \quad-\left\|\tilde{x}_{n}-u_{n}+(p-q)\right\|^{2} \\
& \quad+2 \mu_{1}\left\|\tilde{x}_{n}-u_{n}+(p-q)\right\| \\
& \quad \times\left\|B_{1} \tilde{x}_{n}-B_{1} q\right\|+2 \lambda_{n} \alpha_{n}\|p\|\left\|\tilde{u}_{n}-p\right\| \\
& \left.\left.+2 \bar{u}_{n}-\tilde{u}_{n}\|\| \bar{u}_{n}-p \|\right]\right\}
\end{aligned}
$$

$$
\begin{aligned}
- & \beta_{n}\left(1-\beta_{n}\right) \\
\times & \left\|\frac{1}{1-\beta_{n}}\left[\gamma_{n}\left(x_{n}-y_{n}\right)+\delta_{n}\left(x_{n}-S y_{n}\right)\right]\right\|^{2} \\
\leq & \left\|x_{n}-p\right\|^{2}-\left(1-\beta_{n}\right)\left(1-\sigma_{n}\right) \\
& \times\left(\left\|x_{n}-\tilde{x}_{n}-(p-q)\right\|^{2}+\left\|\tilde{x}_{n}-u_{n}+(p-q)\right\|^{2}\right) \\
& +2 \mu_{2}\left\|x_{n}-\tilde{x}_{n}-(p-q)\right\|\left\|B_{2} x_{n}-B_{2} p\right\| \\
& +2 \mu_{1}\left\|\tilde{x}_{n}-u_{n}+(p-q)\right\|\left\|B_{1} \tilde{x}_{n}-B_{1} q\right\| \\
& +2 \lambda_{n} \alpha_{n}\|p\|\left\|\widetilde{u}_{n}-p\right\|+2\left\|\bar{u}_{n}-\widetilde{u}_{n}\right\|\left\|\bar{u}_{n}-p\right\| \\
& -\beta_{n}\left(1-\beta_{n}\right)\left\|\frac{1}{1-\beta_{n}}\left[\gamma_{n}\left(x_{n}-y_{n}\right)+\delta_{n}\left(x_{n}-S y_{n}\right)\right]\right\|^{2},
\end{aligned}
$$

which hence implies that

$$
\begin{aligned}
& \left(1-\beta_{n}\right)\left(1-\sigma_{n}\right) \\
& \quad \times\left(\left\|x_{n}-\tilde{x}_{n}-(p-q)\right\|^{2}+\left\|\tilde{x}_{n}-u_{n}+(p-q)\right\|^{2}\right) \\
& \quad+\beta_{n}\left(1-\beta_{n}\right)\left\|\frac{1}{1-\beta_{n}}\left[\gamma_{n}\left(x_{n}-y_{n}\right)+\delta_{n}\left(x_{n}-S y_{n}\right)\right]\right\|^{2}
\end{aligned}
$$

$$
\begin{aligned}
\leq & \left\|x_{n}-p\right\|^{2}-\left\|x_{n+1}-p\right\|^{2}+2 \lambda_{n} \alpha_{n}\|p\|\left\|\tilde{u}_{n}-p\right\| \\
& +2 \mu_{2}\left\|x_{n}-\tilde{x}_{n}-(p-q)\right\|\left\|B_{2} x_{n}-B_{2} p\right\| \\
& +2 \mu_{1}\left\|\tilde{x}_{n}-u_{n}+(p-q)\right\|\left\|B_{1} \tilde{x}_{n}-B_{1} q\right\| \\
& +2\left\|\bar{u}_{n}-\tilde{u}_{n}\right\|\left\|\bar{u}_{n}-p\right\| .
\end{aligned}
$$

Since $0<\liminf _{n \rightarrow \infty} \beta_{n} \leq \limsup _{n \rightarrow \infty} \beta_{n}<1$, $\limsup _{n \rightarrow \infty} \sigma_{n}<1,\left\{\lambda_{n}\right\} \subset[a, b], \alpha_{n} \rightarrow 0,\left\|B_{2} x_{n}-B_{2} p\right\| \rightarrow$ $0,\left\|B_{1} \widetilde{x}_{n}-B_{1} q\right\| \rightarrow 0,\left\|\bar{u}_{n}-\widetilde{u}_{n}\right\| \rightarrow 0$ and $\lim _{n \rightarrow \infty}\left\|x_{n}-p\right\|$ exists, it follows from the boundedness of $\left\{x_{n}\right\},\left\{\tilde{x}_{n}\right\},\left\{u_{n}\right\},\left\{\tilde{u}_{n}\right\}$ and $\left\{\bar{u}_{n}\right\}$ that $\lim _{n \rightarrow \infty}\left\|x_{n}-\tilde{x}_{n}-(p-q)\right\|=0$ :

$$
\begin{gathered}
\lim _{n \rightarrow \infty}\left\|\tilde{x}_{n}-u_{n}+(p-q)\right\|=0, \\
\lim _{n \rightarrow \infty}\left\|\gamma_{n}\left(x_{n}-y_{n}\right)+\delta_{n}\left(x_{n}-S y_{n}\right)\right\|=0 .
\end{gathered}
$$

Consequently, it immediately follows that

$$
\lim _{n \rightarrow \infty}\left\|x_{n}-u_{n}\right\|=0, \quad \lim _{n \rightarrow \infty}\left\|x_{n}-\bar{u}_{n}\right\|=0 .
$$

This together with $\left\|y_{n}-\bar{u}_{n}\right\| \leq \sigma_{n}\left\|x_{n}-\bar{u}_{n}\right\| \rightarrow 0$ implies that

$$
\lim _{n \rightarrow \infty}\left\|x_{n}-y_{n}\right\|=0 \text {. }
$$

Since

$$
\begin{aligned}
\left\|\delta_{n}\left(S y_{n}-x_{n}\right)\right\| \\
\quad=\left\|\gamma_{n}\left(x_{n}-y_{n}\right)+\delta_{n}\left(x_{n}-S y_{n}\right)+\gamma_{n}\left(y_{n}-x_{n}\right)\right\| \\
\quad \leq\left\|\gamma_{n}\left(x_{n}-y_{n}\right)+\delta_{n}\left(x_{n}-S y_{n}\right)\right\|+\gamma_{n}\left\|x_{n}-y_{n}\right\|,
\end{aligned}
$$

we have

$$
\lim _{n \rightarrow \infty}\left\|S y_{n}-x_{n}\right\|=0, \quad \lim _{n \rightarrow \infty}\left\|S y_{n}-y_{n}\right\|=0 .
$$

Step 4. $\left\{x_{n}\right\},\left\{u_{n}\right\}$ and $\left\{\tilde{u}_{n}\right\}$ converge weakly to an element $\bar{x} \in$ $\operatorname{Fix}(S) \cap \Xi \cap \Gamma$.

Indeed, since $\left\{x_{n}\right\}$ is bounded, there exists a subsequence $\left\{x_{n_{i}}\right\}$ of $\left\{x_{n}\right\}$ that converges weakly to some $\bar{x} \in C$. We obtain that $\bar{x} \in \operatorname{Fix}(S) \cap \Xi \cap \Gamma$. Taking into account that $\left\|x_{n}-u_{n}\right\| \rightarrow 0$ and $\left\|\tilde{u}_{n}-u_{n}\right\| \rightarrow 0$ and $\left\|x_{n}-y_{n}\right\| \rightarrow 0$, we deduce that $y_{n_{i}} \rightarrow \bar{x}$ weakly and $\tilde{u}_{n_{i}} \rightarrow \bar{x}$ weakly.

First, it is clear from Lemma 15 and $\left\|S y_{n}-y_{n}\right\| \rightarrow 0$ that $\bar{x} \in \operatorname{Fix}(S)$. Now let us show that $\bar{x} \in \Xi$. Note that

$$
\begin{aligned}
\left\|x_{n}-G\left(x_{n}\right)\right\| & \\
=\| x_{n}-P_{C}[ & P_{C}\left(x_{n}-\mu_{2} B_{2} x_{n}\right) \\
& \left.\quad-\mu_{1} B_{1} P_{C}\left(x_{n}-\mu_{2} B_{2} x_{n}\right)\right] \| \\
=\left\|x_{n}-u_{n}\right\| & \longrightarrow 0,
\end{aligned}
$$

as $n \rightarrow \infty$, where $G: C \rightarrow C$ is defined as that in Lemma 1 . According to Lemma 15, we get $\bar{x} \in \Xi$. Further, let us show that $\bar{x} \in \Gamma$. As a matter of fact, define

$$
T v= \begin{cases}\nabla f(v)+N_{C} v, & \text { if } v \in C, \\ \emptyset, & \text { if } v \notin C,\end{cases}
$$


where $N_{C} v=\left\{w \in \mathscr{H}_{1}:\langle v-u, w\rangle \geq 0, \forall u \in C\right\}$. Utilizing the argument similar to that of Step 4 in the proof of Theorem 20, from the relation

$$
\tilde{u}_{n}=P_{C}\left(u_{n}-\lambda_{n} \nabla f_{\alpha_{n}}\left(u_{n}\right)\right), \quad v \in C,
$$

we can easily conclude that

$$
\langle v-\bar{x}, w\rangle \geq 0 \text {. }
$$

It is easy to see that $\bar{x} \in \Gamma$. Therefore, $\bar{x} \in \operatorname{Fix}(S) \cap \Xi \cap \Gamma$. Finally, utilizing the Opial condition [41], we infer that $\left\{x_{n}\right\}$ converges weakly to $\bar{x} \in \operatorname{Fix}(S) \cap \Xi \cap \Gamma$. Further, from $\left\|x_{n}-u_{n}\right\| \rightarrow 0$ and $\left\|x_{n}-\widetilde{u}_{n}\right\| \rightarrow 0$, it follows that both $\left\{u_{n}\right\}$ and $\left\{\widetilde{u}_{n}\right\}$ converge weakly to $\bar{x}$. This completes the proof.

Corollary 24. Let $C$ be a nonempty closed convex subset of a real Hilbert space $\mathscr{H}_{1}$. Let $A \in B\left(\mathscr{H}_{1}, \mathscr{H}_{2}\right)$ and $B_{i}: C \rightarrow \mathscr{H}_{1}$ be $\beta_{i}$-inverse strongly monotone for $i=1,2$. Let $S: C \rightarrow C$ be a $k$-strictly pseudocontractive mapping such that $\operatorname{Fix}(S) \cap \Xi \cap$ $\Gamma \neq \emptyset$. For given $x_{0} \in C$ arbitrarily, let the sequences $\left\{x_{n}\right\},\left\{u_{n}\right\}$, $\left\{\widetilde{u}_{n}\right\}$ be generated iteratively by

$$
\begin{gathered}
u_{n}=P_{C}\left[P_{C}\left(x_{n}-\mu_{2} B_{2} x_{n}\right)-\mu_{1} B_{1} P_{C}\left(x_{n}-\mu_{2} B_{2} x_{n}\right)\right], \\
\tilde{u}_{n}=P_{C}\left(u_{n}-\lambda_{n} \nabla f_{\alpha_{n}}\left(u_{n}\right)\right), \\
y_{n}=P_{C}\left(u_{n}-\lambda_{n} \nabla f_{\alpha_{n}}\left(\tilde{u}_{n}\right)\right), \\
x_{n+1}=\beta_{n} x_{n}+\gamma_{n} y_{n}+\delta_{n} S y_{n}, \quad \forall n \geq 0,
\end{gathered}
$$

where $\mu_{i} \in\left(0,2 \beta_{i}\right)$ for $i=1,2,\left\{\alpha_{n}\right\} \subset(0, \infty),\left\{\lambda_{n}\right\} \subset$ $\left(0,1 /\|A\|^{2}\right)$ and $\left\{\beta_{n}\right\},\left\{\gamma_{n}\right\},\left\{\delta_{n}\right\} \subset[0,1]$ such that

(i) $\sum_{n=0}^{\infty} \alpha_{n}<\infty$;

(ii) $\beta_{n}+\gamma_{n}+\delta_{n}=1$ and $\left(\gamma_{n}+\delta_{n}\right) k \leq \gamma_{n}$ for all $n \geq 0$;

(iii) $0<\liminf _{n \rightarrow \infty} \beta_{n} \leq \limsup _{n \rightarrow \infty} \beta_{n}<1$ and $\liminf _{n \rightarrow \infty} \delta_{n}>0$;

(iv) $0<\liminf _{n \rightarrow \infty} \lambda_{n} \leq \lim \sup _{n \rightarrow \infty} \lambda_{n}<1 /\|A\|^{2}$.

Then the sequences $\left\{x_{n}\right\},\left\{u_{n}\right\},\left\{\tilde{u}_{n}\right\}$ converge weakly to an element $\bar{x} \in \operatorname{Fix}(S) \cap \Xi \cap \Gamma$. Furthermore, $(\bar{x}, \bar{y})$ is a solution of GSVI (3), where $\bar{y}=P_{C}\left(\bar{x}-\mu_{2} B_{2} \bar{x}\right)$.

Next, utilizing Corollary 24, we derive the following result.

Corollary 25. Let $C$ be a nonempty closed convex subset of a real Hilbert space $\mathscr{H}_{1}$. Let $A \in B\left(\mathscr{H}_{1}, \mathscr{H}_{2}\right)$ and $S: C \rightarrow C$ be a nonexpansive mapping such that $\operatorname{Fix}(S) \cap \Gamma \neq \emptyset$. For given $x_{0} \in C$ arbitrarily, let the sequences $\left\{x_{n}\right\},\left\{\tilde{u}_{n}\right\}$ be generated iteratively by

$$
\begin{aligned}
& \tilde{u}_{n}=P_{C}\left(x_{n}-\lambda_{n} \nabla f_{\alpha_{n}}\left(x_{n}\right)\right), \\
& x_{n+1}=\beta_{n} x_{n}+\left(1-\beta_{n}\right) S P_{C}\left(x_{n}-\lambda_{n} \nabla f_{\alpha_{n}}\left(\tilde{u}_{n}\right)\right),
\end{aligned}
$$

$\forall n \geq 0$,

where $\left\{\alpha_{n}\right\} \subset(0, \infty),\left\{\lambda_{n}\right\} \subset\left(0,1 /\|A\|^{2}\right)$ and $\left\{\beta_{n}\right\} \subset[0,1]$ such that (i) $\sum_{n=0}^{\infty} \alpha_{n}<\infty$;

(ii) $0<\liminf _{n \rightarrow \infty} \beta_{n} \leq \lim \sup _{n \rightarrow \infty} \beta_{n}<1$;

(iii) $0<\liminf _{n \rightarrow \infty} \lambda_{n} \leq \lim \sup _{n \rightarrow \infty} \lambda_{n}<1 /\|A\|^{2}$.

Then, both the sequences $\left\{x_{n}\right\}$ and $\left\{\tilde{u}_{n}\right\}$ converge weakly to an element $\bar{x} \in \operatorname{Fix}(S) \cap \Gamma$.

Proof. In Corollary 24, put $B_{1}=B_{2}=0$ and $\gamma_{n}=0$. Then, $\Xi=C, \beta_{n}+\delta_{n}=1$ for all $n \geq 0$, and the iterative scheme (114) is equivalent to

$$
\begin{gathered}
u_{n}=x_{n}, \\
\tilde{u}_{n}=P_{C}\left(u_{n}-\lambda_{n} \nabla f_{\alpha_{n}}\left(u_{n}\right)\right), \\
y_{n}=P_{C}\left(u_{n}-\lambda_{n} \nabla f_{\alpha_{n}}\left(\tilde{u}_{n}\right)\right), \\
x_{n+1}=\beta_{n} x_{n}+\delta_{n} S y_{n}, \quad \forall n \geq 0 .
\end{gathered}
$$

This is equivalent to (115). Since $S$ is a nonexpansive mapping, $S$ must be a $k$-strictly pseudocontractive mapping with $k=0$. In this case, it is easy to see that all the conditions (i)-(iv) in Corollary 24 are satisfied. Therefore, in terms of Corollary 24, we obtain the desired result.

Remark 26. Compared with the Ceng and Yao [31, Theorem 3.1], our Corollary 25 coincides essentially with [31, Theorem 3.1]. This shows that our Theorem 23 includes [31, Theorem 3.1] as a special case.

Remark 27. Our Theorems 20 and 23 improve, extend, and develop [20, Theorem 5.7], [31, Theorem 3.1], [7, Theorem $3.2]$, and [14, Theorem 3.1] in the following aspects.

(i) Compared with the relaxed extragradient iterative algorithm in [7, Theorem 3.2], our Mann-type extragradient iterative algorithms with regularization remove the requirement of boundedness for the domain $C$ in which various mappings are defined.

(ii) Because [31, Theorem 3.1] is the supplementation, improvement, and extension of [20, Theorem 5.7] and our Theorem 23 includes [31, Theorem 3.1] as a special case, beyond question our results are very interesting and quite valuable.

(iii) The problem of finding an element of $\operatorname{Fix}(S) \cap \Xi \cap \Gamma$ in our Theorems 20 and 23 is more general than the corresponding problems in [20, Theorem 5.7] and [31, Theorem 3.1], respectively.

(iv) The hybrid extragradient method for finding an element of $\operatorname{Fix}(S) \cap \Xi \cap \operatorname{VI}(C, A)$ in [14, Theorem 3.1] is extended to develop our Mann-type extragradient iterative algorithms (16) and (17) with regularization for finding an element of $\operatorname{Fix}(S) \cap \Xi \cap \Gamma$.

(v) The proof of our results are very different from that of [14, Theorem 3.1] because our argument technique depends on the Opial condition, the restriction on the regularization parameter sequence $\left\{\alpha_{n}\right\}$, and the properties of the averaged mappings $P_{C}\left(I-\lambda_{n} \nabla f_{\alpha_{n}}\right)$ to a great extent. 
(vi) Because our iterative algorithms (16) and (17) involve two inverse strongly monotone mappings $B_{1}$ and $B_{2}$, a $k$-strictly pseudocontractive self-mapping $S$ and several parameter sequences, they are more flexible and more subtle than the corresponding ones in [20, Theorem 5.7] and [31, Theorem 3.1], respectively.

\section{Acknowledgments}

In this research, the first author was partially supported by the National Science Foundation of China (11071169) and Ph.D. Program Foundation of Ministry of Education of China (20123127110002). The third author was partially supported by Grant NSC 101-2115-M-037-001.

\section{References}

[1] A. Bnouhachem, M. Aslam Noor, and Z. Hao, "Some new extragradient iterative methods for variational inequalities," Nonlinear Analysis. Theory, Methods \& Applications, vol. 70, no. 3, pp. 1321-1329, 2009.

[2] L.-C. Ceng, Q. H. Ansari, and J.-C. Yao, "Viscosity approximation methods for generalized equilibrium problems and fixed point problems," Journal of Global Optimization, vol. 43, no. 4, pp. 487-502, 2009.

[3] L.-C. Ceng and S. Huang, "Modified extragradient methods for strict pseudo-contractions and monotone mappings," Taiwanese Journal of Mathematics, vol. 13, no. 4, pp. 1197-1211, 2009.

[4] L.-C. Ceng, C.-Y. Wang, and J.-C. Yao, "Strong convergence theorems by a relaxed extragradient method for a general system of variational inequalities," Mathematical Methods of Operations Research, vol. 67, no. 3, pp. 375-390, 2008.

[5] L.-C. Ceng and J.-C. Yao, "An extragradient-like approximation method for variational inequality problems and fixed point problems," Applied Mathematics and Computation, vol. 190, no. 1, pp. 205-215, 2007.

[6] L.-C. Ceng and J.-C. Yao, "Relaxed viscosity approximation methods for fixed point problems and variational inequality problems," Nonlinear Analysis: Theory, Methods \& Applications, vol. 69, no. 10, pp. 3299-3309, 2008.

[7] Y. Yao, Y.-C. Liou, and S. M. Kang, "Approach to common elements of variational inequality problems and fixed point problems via a relaxed extragradient method," Computers \& Mathematics with Applications, vol. 59, no. 11, pp. 3472-3480, 2010.

[8] L.-C. Zeng and J.-C. Yao, "Strong convergence theorem by an extragradient method for fixed point problems and variational inequality problems," Taiwanese Journal of Mathematics, vol. 10, no. 5, pp. 1293-1303, 2006.

[9] N. Nadezhkina and W. Takahashi, "Strong convergence theorem by a hybrid method for nonexpansive mappings and Lipschitz-continuous monotone mappings," SIAM Journal on Optimization, vol. 16, no. 4, pp. 1230-1241, 2006.

[10] W. Takahashi and M. Toyoda, "Weak convergence theorems for nonexpansive mappings and monotone mappings," Journal of Optimization Theory and Applications, vol. 118, no. 2, pp. 417428, 2003.

[11] G. M. Korpelevič, "An extragradient method for finding saddle points and for other problems," Ekonomika i Matematicheskie Metody, vol. 12, no. 4, pp. 747-756, 1976.

[12] Y. Yao and J.-C. Yao, "On modified iterative method for nonexpansive mappings and monotone mappings," Applied
Mathematics and Computation, vol. 186, no. 2, pp. 1551-1558, 2007.

[13] R. U. Verma, "On a new system of nonlinear variational inequalities and associated iterative algorithms," Mathematical Sciences Research Hot-Line, vol. 3, no. 8, pp. 65-68, 1999.

[14] L.-C. Ceng, S.-M. Guu, and J.-C. Yao, "Finding common solutions of a variational inequality, a general system of variational inequalities, and a fixed-point problem via a hybrid extragradient method," Fixed Point Theory and Applications, vol. 2011, Article ID 626159, 22 pages, 2011.

[15] Y. Censor and T. Elfving, "A multiprojection algorithm using Bregman projections in a product space," Numerical Algorithms, vol. 8, no. 2-4, pp. 221-239, 1994.

[16] C. Byrne, "Iterative oblique projection onto convex sets and the split feasibility problem," Inverse Problems, vol. 18, no. 2, pp. 441453, 2002.

[17] Y. Censor, T. Bortfeld, B. Martin, and A. Trofimov, "A unified approach for inversion problems in intensity-modulated radiation therapy," Physics in Medicine \& Biology, vol. 51, no. 10, pp. 2353-2365, 2006.

[18] Y. Censor, T. Elfving, N. Kopf, and T. Bortfeld, “The multiplesets split feasibility problem and its applications for inverse problems," Inverse Problems, vol. 21, no. 6, pp. 2071-2084, 2005.

[19] Y. Censor, A. Motova, and A. Segal, "Perturbed projections and subgradient projections for the multiple-sets split feasibility problem," Journal of Mathematical Analysis and Applications, vol. 327, no. 2, pp. 1244-1256, 2007.

[20] H.-K. Xu, "Iterative methods for the split feasibility problem in infinite-dimensional Hilbert spaces," Inverse Problems, vol. 26, no. 10, Article ID 105018, 17 pages, 2010.

[21] C. Byrne, "A unified treatment of some iterative algorithms in signal processing and image reconstruction," Inverse Problems, vol. 20, no. 1, pp. 103-120, 2004.

[22] B. Qu and N. Xiu, "A note on the CQ algorithm for the split feasibility problem," Inverse Problems, vol. 21, no. 5, pp. 1655$1665,2005$.

[23] H.-K. Xu, "A variable Krasnosel'skii-Mann algorithm and the multiple-set split feasibility problem," Inverse Problems, vol. 22, no. 6, pp. 2021-2034, 2006.

[24] Q. Yang, "The relaxed CQ algorithm solving the split feasibility problem," Inverse Problems, vol. 20, no. 4, pp. 1261-1266, 2004.

[25] J. Zhao and Q. Yang, "Several solution methods for the split feasibility problem," Inverse Problems, vol. 21, no. 5, pp. 17911799, 2005.

[26] M. I. Sezan and H. Stark, "Applications of convex projection theory to image recovery in tomography and related areas," in Image Recovery Theory and Applications, H. Stark, Ed., pp. 415462, Academic Press, Orlando, Fla, USA, 1987.

[27] B. Eicke, "Iteration methods for convexly constrained ill-posed problems in Hilbert space," Numerical Functional Analysis and Optimization, vol. 13, no. 5-6, pp. 413-429, 1992.

[28] L. Landweber, "An iteration formula for Fredholm integral equations of the first kind," American Journal of Mathematics, vol. 73, pp. 615-624, 1951.

[29] L. C. Potter and K. S. Arun, "A dual approach to linear inverse problems with convex constraints," SIAM Journal on Control and Optimization, vol. 31, no. 4, pp. 1080-1092, 1993.

[30] P. L. Combettes and V. R. Wajs, "Signal recovery by proximal forward-backward splitting," Multiscale Modeling \& Simulation, vol. 4, no. 4, pp. 1168-1200, 2005. 
[31] L.-C. Ceng, Q. H. Ansari, and J.-C. Yao, "An extragradient method for solving split feasibility and fixed point problems," Computers \& Mathematics with Applications, vol. 64, no. 4, pp. 633-642, 2012.

[32] N. Nadezhkina and W. Takahashi, "Weak convergence theorem by an extragradient method for nonexpansive mappings and monotone mappings," Journal of Optimization Theory and Applications, vol. 128, no. 1, pp. 191-201, 2006.

[33] D. P. Bertsekas and E. M. Gafni, "Projection methods for variational inequalities with application to the traffic assignment problem," Mathematical Programming Study, vol. 17, pp. 139-159, 1982.

[34] D. Han and H. K. Lo, "Solving non-additive traffic assignment problems: a descent method for co-coercive variational inequalities," European Journal of Operational Research, vol. 159, no. 3, pp. 529-544, 2004.

[35] P. L. Combettes, "Solving monotone inclusions via compositions of nonexpansive averaged operators," Optimization, vol. 53, no. 5-6, pp. 475-504, 2004.

[36] K. Goebel and W. A. Kirk, Topics in Metric Fixed Point Theory, vol. 28 of Cambridge Studies in Advanced Mathematics, Cambridge University Press, 1990.

[37] G. Marino and H.-K. Xu, "Weak and strong convergence theorems for strict pseudo-contractions in Hilbert spaces," Journal of Mathematical Analysis and Applications, vol. 329, no. 1, pp. 336-346, 2007.

[38] M. O. Osilike, S. C. Aniagbosor, and B. G. Akuchu, "Fixed points of asymptotically demicontractive mappings in arbitrary Banach spaces," Panamerican Mathematical Journal, vol. 12, no. 2, pp. 77-88, 2002.

[39] K.-K. Tan and H. K. Xu, "Approximating fixed points of nonexpansive mappings by the Ishikawa iteration process," Journal of Mathematical Analysis and Applications, vol. 178, no. 2, pp. 301-308, 1993.

[40] R. T. Rockafellar, "On the maximality of sums of nonlinear monotone operators," Transactions of the American Mathematical Society, vol. 149, pp. 75-88, 1970.

[41] Z. Opial, "Weak convergence of the sequence of successive approximations for nonexpansive mappings," Bulletin of the American Mathematical Society, vol. 73, pp. 591-597, 1967. 


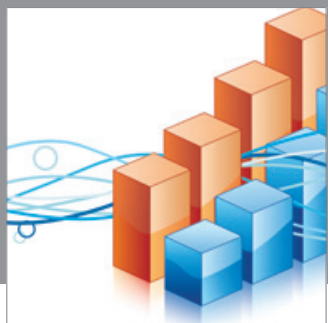

Advances in

Operations Research

mansans

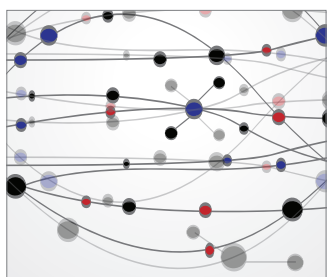

The Scientific World Journal
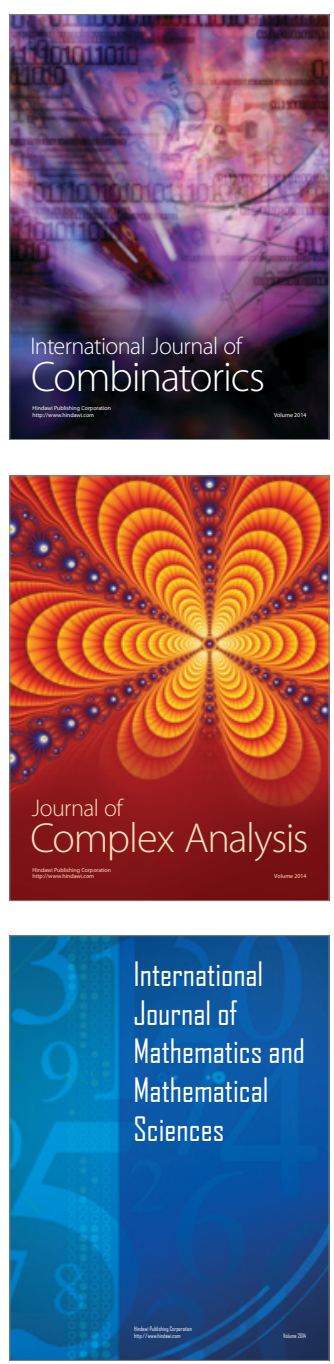
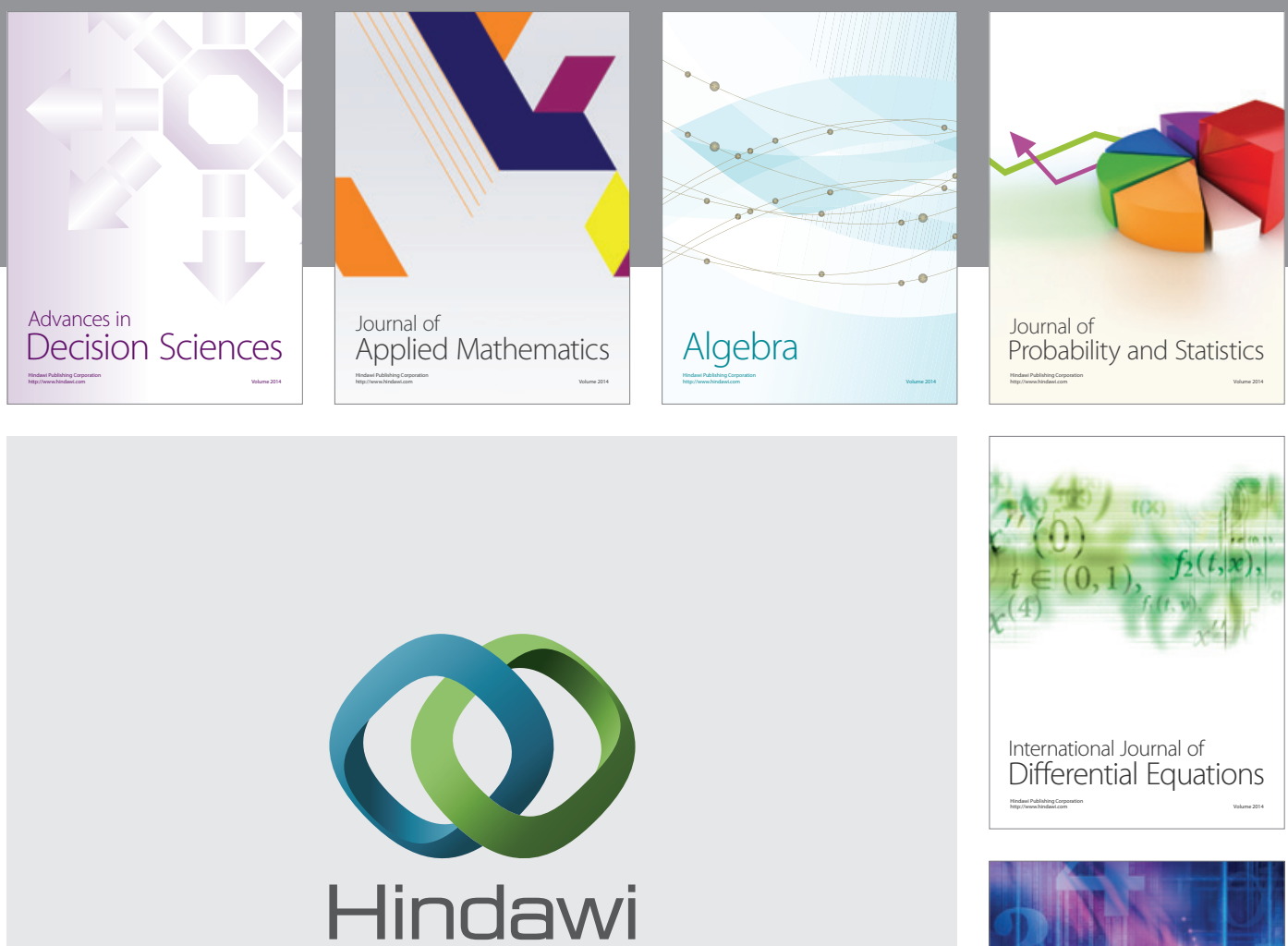

Submit your manuscripts at http://www.hindawi.com
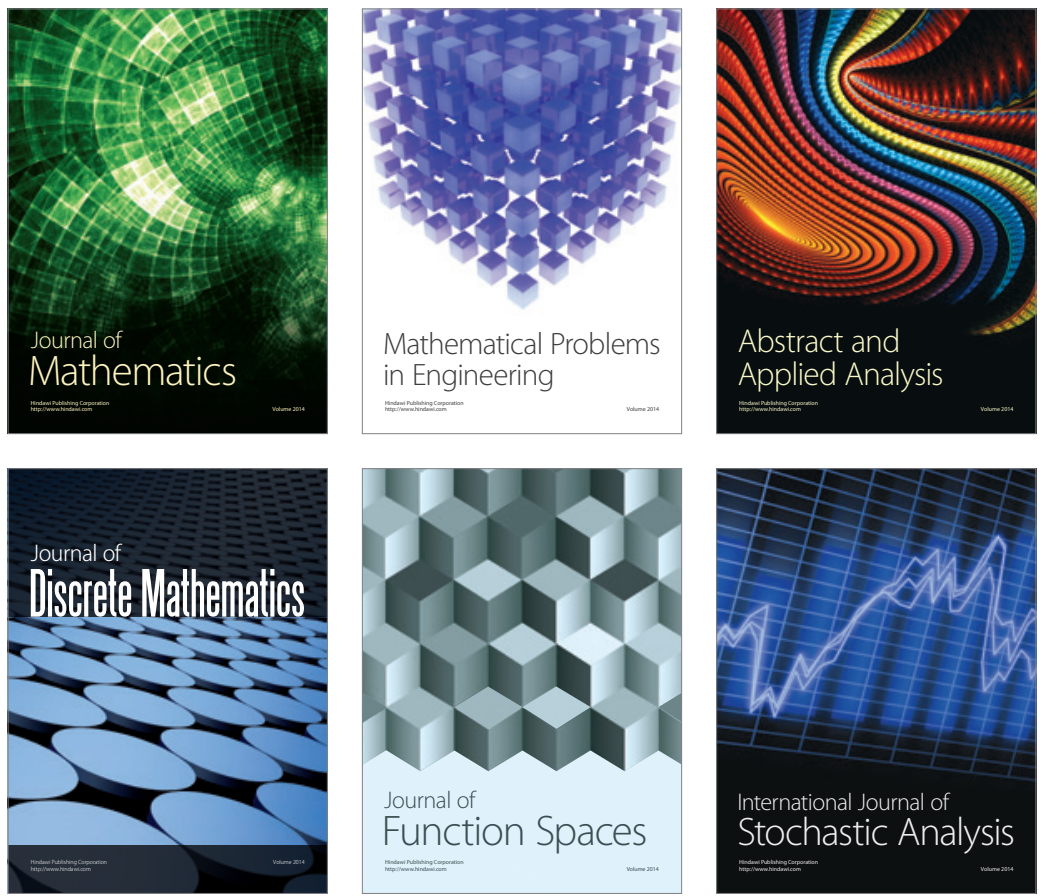

Journal of

Function Spaces

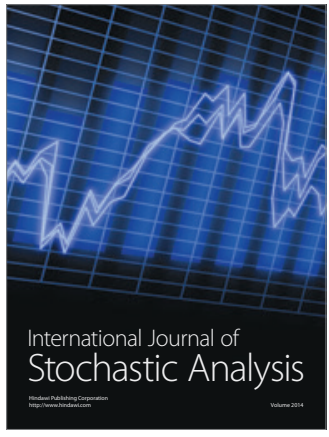

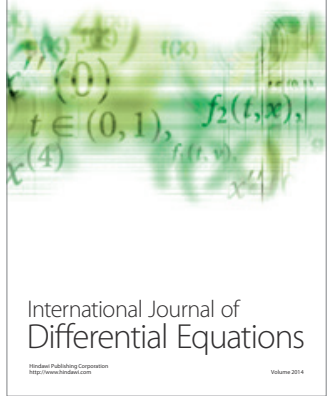
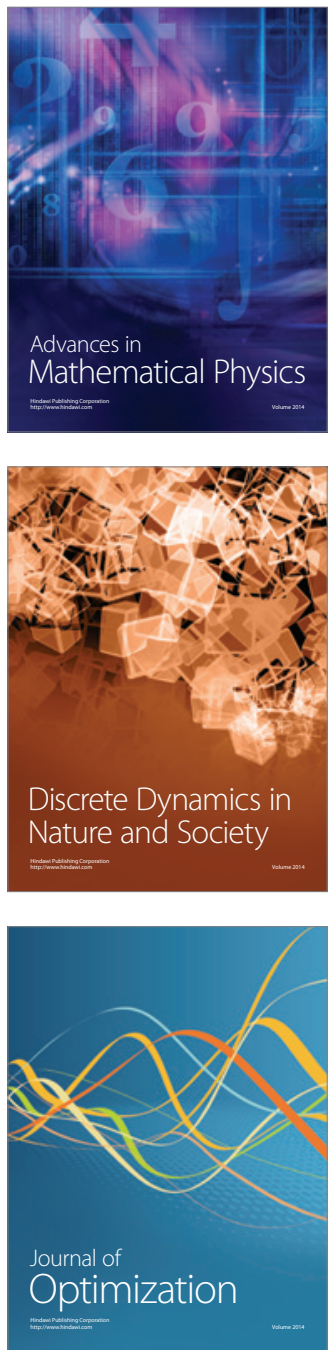